\title{
The uncharacterized protein FAM47E interacts with PRMT5 and regulates its functions
}

\author{
Baskar Chakrapani ${ }^{1, \star}$, Mohd Imran K Khan ${ }^{1, \star}$, Rajashekar Varma Kadumuri ${ }^{2}$, Somlee Gupta ${ }^{1}$, Mamta Verma , \\ Sharad Awasthi ${ }^{1}$, Gayathri Govindaraju ${ }^{3}$, Arun Mahesh ${ }^{1}$, Arumugam Rajavelu ${ }^{3}$, Sreenivas Chavali ${ }^{2}$, \\ Arunkumar Dhayalan ${ }^{1}$ (D)
}

Protein arginine methyltransferase 5 (PRMT5) symmetrically dimethylates arginine residues in various proteins affecting diverse cellular processes such as transcriptional regulation, splicing, DNA repair, differentiation, and cell cycle. Elevated levels of PRMT5 are observed in several types of cancers and are associated with poor clinical outcomes, making PRMT5 an important diagnostic marker and/or therapeutic target for cancers. Here, using yeast two-hybrid screening, followed by immunoprecipitation and pull-down assays, we identify a previously uncharacterized protein, FAM47E, as an interaction partner of PRMT5. We report that FAM47E regulates steady-state levels of PRMT5 by affecting its stability through inhibition of its proteasomal degradation. Importantly, FAM47E enhances the chromatin association and histone methylation activity of PRMT5. The PRMT5-FAM47E interaction affects the regulation of PRMT5 target genes expression and colony-forming capacity of the cells. Taken together, we identify FAM47E as a protein regulator of PRMT5, which promotes the functions of this versatile enzyme. These findings imply that disruption of PRMT5-FAM47E interaction by small molecules might be an alternative strategy to attenuate the oncogenic function(s) of PRMT5.

DOI 10.26508/ Isa.202000699 | Received 13 March 2020 | Revised 18 December 2020 | Accepted 18 December 2020 | Published online 29 December 2020

\section{Introduction}

Arginine methylation is a widely prevalent, important posttranslational modification affecting various cellular processes (Peng \& Wong, 2017). Protein arginine methyltransferase 5 (PRMT5) belongs to type II methyltransferases that symmetrically dimethylate the arginine residues of the target proteins (Bedford \& Clarke, 2009). PRMT5 plays an important role in the regulation of gene expression, splicing, chromatin remodeling, cell differentiation, and development (Stopa et al, 2015). PRMT5 participates in epigenetic regulation of chromatin structure and gene expression by introducing symmetric dimethylation at arginine 3 of histone 4 (H4R3me2s), arginine 2 and 8 of histone 3 (H3R2me2s and H3R8me2s) and arginine 3 of histone 2A (H2AR3me2s) (Pollack et al, 1999; Branscombe et al, 2001; Pal et al, 2004; Ancelin et al, 2006; Migliori et al, 2012). Apart from histones, PRMT5 methylates and regulates the function of a wide variety of non-histone proteins involved in diverse biological processes such as (i) DNA repair: FEN1 (Guo et al, 2010); (ii) transcription: p53 (Jansson et al, 2008; Scoumanne et al, 2009), SPT5 (Kwak et al, 2003), E2F1 (Cho et al, 2012), MBD2 (Tan \& Nakielny, 2006), HOXA9 (Bandyopadhyay et al, 2012), NF-KB (Harris et al, 2016), SREBP1 (Liu et al, 2016), FOXP3 (Nagai et al, 2019), BCL6 (Lu et al, 2018), Tip60 (Clarke et al, 2017), and RNAPII (Zhao et al, 2016); (iii) splicing: Sm proteins (Friesen et al, 2001; Meister et al, 2001), (iv) translation: ribosomal protein S10 (Ren et al, 2010) and hnRNP A1 (Gao et al, 2017), (v) signaling: EGFR (Hsu et al, 2011), PDGFRa (Calabretta et al, 2018), and CRAF (Andreu-Perez et al, 2011); (vi) organelle biogenesis: GM130 (Zhou et al, 2010); and (vii) stress response: G3BP1 (Tsai et al, 2016) and LSM4 (Arribas-Layton et al, 2016).

PRMT5 plays a critical role in the differentiation of primordial germ cells, nerve cells, myocytes, and keratinocytes (Ancelin et al, 2006; Dacwag et al, 2007, 2009; Huang et al, 2011; Chittka et al, 2012; Kanade \& Eckert, 2012; Paul et al, 2012). Notably, the knockout of PRMT5 leads to embryonic lethality, reflecting its essentiality for development and survival (Tee et al, 2010). From a pathological stand point, aberrant expression of human PRMT5 is observed in diverse cancer types (Stopa et al, 2015; Xiao et al, 2019). Elevated expression of PRMT5 in epithelial ovarian cancer and non-small cell lung cancer is associated with poor clinical outcomes and patient survival (Bao et al, 2013; Györffy et al, 2013; Stopa et al, 2015). Depletion of PRMT5 inhibits cell proliferation, clonogenic capacity of the cells, and improves the prognosis of cancer patients making PRMT5 an important target for cancer therapy (Pal et al, 2004; Scoumanne et al, 2009; Wei et al, 2012; Chung et al, 2013; Morettin et al, 2015; Yang et al, 2016; Banasavadi-Siddegowda et al, 2018; Saloura et al, 2018; Xiao et al, 2019).

${ }^{1}$ Department of Biotechnology, Pondicherry University, Puducherry, India ${ }^{2}$ Department of Biology, Indian Institute of Science Education and Research (IISER) Tirupati, Tirupati, India ${ }^{3}$ Interdisciplinary Biology, Rajiv Gandhi Centre for Biotechnology, Trivandrum, India 
The enzymatic activity, substrate specificity, subcellular localization, and functions of PRMT5 is often regulated by its interaction partners (Stopa et al, 2015). For instance, PRMT5 forms a heterooctameric complex with WD40 repeat protein, MEP50, and the PRMT5-MEP50 complex has higher enzymatic activity than PRMT5 in the unbound state (Friesen et al, 2002; Antonysamy et al, 2012; Ho et al, 2013). PRMT5 interacts with pICln or RioK1 in a mutually exclusive manner and promotes the methylation of Sm proteins or nucleolin, respectively. This highlights that the interaction partners determine the substrate specificity of PRMT5 (Friesen et al, 2001; Meister et al, 2001; Guderian et al, 2011). Interaction of PRMT5 with Menin or COPR5 promotes the recruitment of PRMT5 to the specific promoter regions of chromatin (Lacroix et al, 2008; Paul et al, 2012; Gurung et al, 2013). Blimp1 interacts with PRMT5 and specifies its sub-cellular localization in primordial germ cells (Ancelin et al, 2006). Binding of PRMT5 to interactors such as AJUBA (Hou et al, 2008), JAK kinase (Pollack et al, 1999; Liu et al, 2011), CRTC2 (Tsai et al, 2013), SHARPIN (Tamiya et al, 2018), carbonic anhydrase 6B (Xu et al, 2017), LYAR (Ju et al, 2014), STRAP (Jansson et al, 2008), PHF1 (Liu et al, 2018), CITED2 (Shin et al, 2018), ERG (Mounir et al, 2016), HSP90 (Maloney et al, 2007), CHIP (Zhang et al, 2016), ZNF224 (Cesaro et al, 2009), and AKT (Zhang et al, 2019) engage PRMT5 in diverse cellular processes.

Given the versatile functions of PRMT5 in the cell and its multiple interaction partners and substrates, the identification and characterization of new interaction partners is very important to obtain a comprehensive understanding of the diverse roles of PRMT5 in the cell. To address this, we performed yeast two-hybrid $(\mathrm{Y} 2 \mathrm{H})$ screening to identify new interaction partners of PRMT5 and identified a novel interaction partner FAM47E (family with sequence similarity 47, member E), an hitherto uncharacterized protein. In addition to identifying the new interaction partner of PRMT5; here, we have characterized the functions of FAM47E. We report that FAM47E regulates the stability, chromatin association and methyltransferase activity of PRMT5, with potential implications in normal physiology and in diseases.

\section{Results}

\section{PRMT5 interacts with FAM47E}

To identify new interaction partners of PRMT5, we performed $\mathrm{Y} 2 \mathrm{H}$ screening of PRMT5 using universal normalized human cDNA library. Three positive clones were obtained in the initial screening with low stringency selection medium which scores for the expression of two reporter genes. Of these three clones, one of them failed in high stringency selection medium which assesses the expression of four reporter genes and hence it was not considered for further study. Sequencing of the other two positive clones revealed that they code for full length COP9 signaling complex subunit 5 (COPS5 protein) and the C-terminal region of FAM47E isoform 2 (43 amino acid to 295 amino acid). The COPS5 protein is known to interact with GAL4 DNA-binding domain directly and produce false positive results in GAL4-based $\mathrm{Y} 2 \mathrm{H}$ screenings (Nordgård et al, 2001; Mohr \& Koegl, 2012) and hence we disregarded it for further consideration. The FAM47E gene has three alternative splice variants, with the isoform 1, the longest being designated as the canonical form in the Uniprot database. However, we obtained FAM47E isoform 2 as putative interaction partner of PRMT5 in our $\mathrm{Y} 2 \mathrm{H}$ screening. Hence, we profiled the expression of all the three FAM47E isoforms in HEK293 cells by quantitative RT ( $q$ RT)-PCR using isoform specific primers. We found that FAM47E isoform 2 mRNA levels are 73 -folds higher than that of FAM47E isoform 1 and it is $\sim 1,218$-folds higher than that of FAM47E isoform 3 suggesting that FAM47E isoform 2 is the predominant isoform in HEK293 cells (Fig S1). Next, we validated PRMT5-FAM47E interaction in $\mathrm{Y} 2 \mathrm{H}$ assay by using full-length FAM47E isoform 2 (hereafter referred as FAM47E) with appropriate vector controls. We observed the expression of reporter genes in both low stringency media and high-stringency media only if the $\mathrm{Y} 2 \mathrm{H}$ constructs of PRMT5 and FAM47E were cotransformed. We could not detect expression of reporter genes when either of the constructs is co-transformed with the corresponding vector control suggesting that FAM47E is a potential interaction partner of PRMT5 (Fig 1A).

To confirm the PRMT5-FAM47E interaction in vivo, we performed the co-immunoprecipitation (Co-IP) experiments by co-expressing Myc-tagged PRMT5 with GFP or GFP-tagged FAM47E in HEK293 cells. Similarly, we co-expressed HA-tagged FAM47E with GFP or GFPtagged PRMT5 for reverse Co-IP experiments. We found that GFPtagged FAM47E efficiently co-precipitated the Myc-tagged PRMT5 in forward Co-IP (Fig 1B) and GFP-tagged PRMT5 co-precipitated the HA-tagged FAM47E in reverse Co-IP (Fig 1C). These observations suggest that PRMT5 could interact with FAM47E in vivo.

We next assessed whether the ectopically expressed GFP-tagged FAM47E could interact with endogenous PRMT5. For this, we overexpressed GFP or GFP-tagged FAM47E in HEK293 cells and performed the Co-IP experiments. We found that GFP-FAM47E efficiently co-precipitated the endogenous PRMT5 suggesting that FAM47E could interact with endogenous levels of PRMT5 (Fig 1D). To assess whether PRMT5 directly interacts with FAM47E, we carried out a GST pull-down assay using recombinant GST-tagged FAM47E and His-tagged PRMT5 proteins. Whereas the control GST protein did not precipitate the His-PRMT5, GST-FAM47E efficiently precipitated His-PRMT5 indicating that PRMT5 interacts with FAM47E directly (Fig 1E). We then performed immunoprecipitation to investigate PRMT5-FAM47E interaction at their endogenous levels in HEK293 cells. Immunoprecipitation experiments were carried out in HEK293 cell lysate using FAM47E antibody or PRMT5 antibody along with the control IgG. We found that immunoprecipitation of FAM47E antibody efficiently co-precipitated the PRMT5 (Fig 1F) and similarly immunoprecipitation of PRMT5 antibody efficiently co-precipitated the FAM47E (Fig 1G). These findings suggest that PRMT5 interacts with FAM47E at their endogenous levels.

Because the WD40 repeat protein, MEP50, interacts and forms a stable hetero-octameric complex with PRMT5 (Friesen et al, 2002; Antonysamy et al, 2012; Ho et al, 2013), we investigated whether FAM47E also interacts with MEP50 in addition to PRMT5. For this, we performed co-immunoprecipitation by co-expressing GFP or GFPFAM47E with Myc-tagged MEP50 in HEK293 cells and found that FAM47E interacts with MEP50 (Fig S2A). This prompted us to investigate if FAM47E affects the binding of MEP50 with PRMT5. For this, we performed co-immunoprecipitation by co-expressing GFP or GFP-PRMT5 and Myc-tagged MEP50 with or without HA tagged 
A

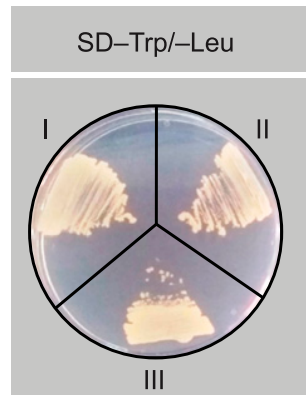

B

SD-Trp/-Leu/-His/-Ade/+Aba/+X-a-Gal

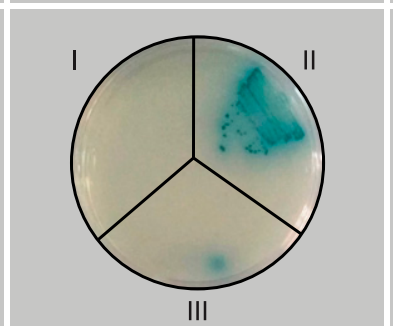

Forward Co-IP: GFP-Trap
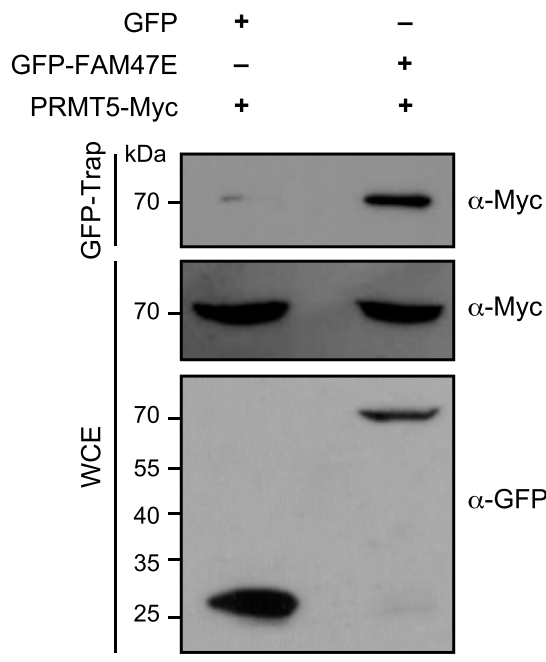

E GST pull down
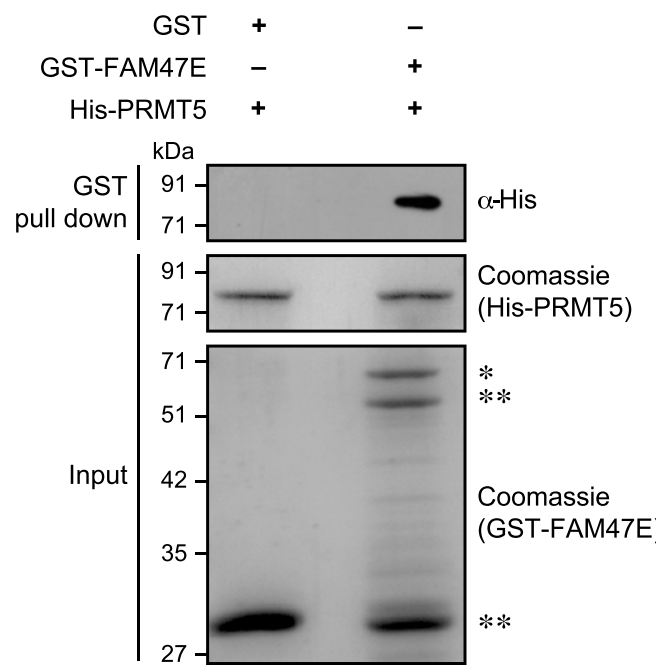

* GST-FAM47E $* *$ Degraded GST-FAM47E

C

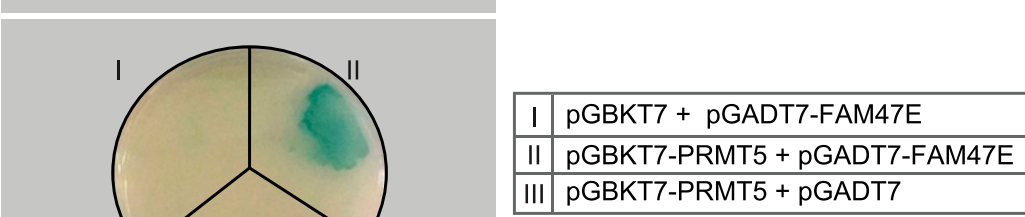

D

IP with endogenous PRMT5: GFP-Trap
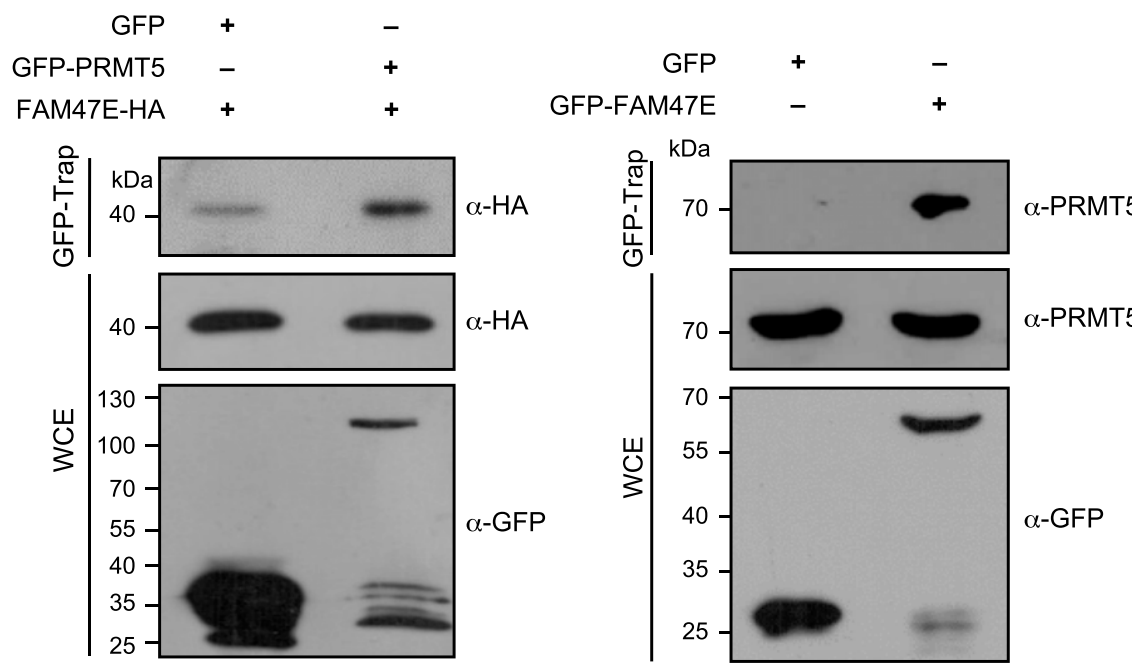

$\mathbf{F}$

G

Endogenous IP: PRMT5
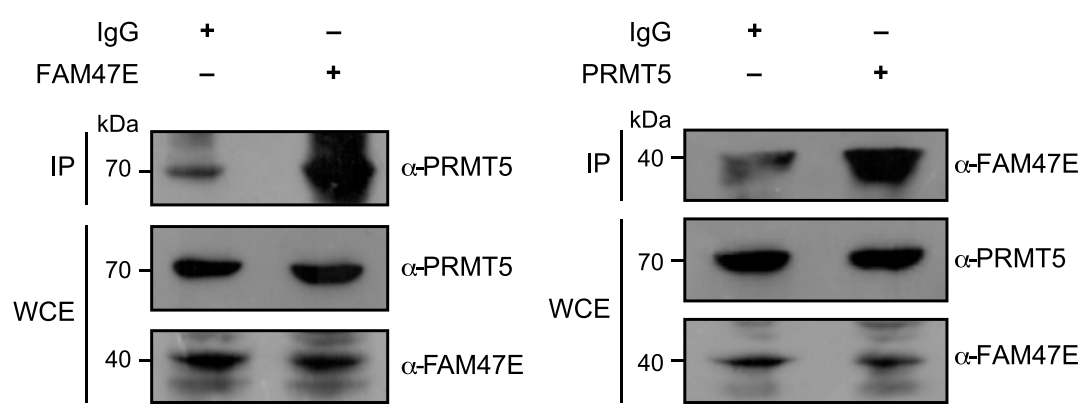

Figure 1. PRMT5 interacts with FAM47E.

(A) $\mathrm{Y} 2 \mathrm{H}$ assay was performed to study the interaction between PRMT5 with FAM47E. The pGBKT7-PRMT5 and PGADT7-FAM47E constructs were allowed to interact with each other or corresponding vector controls. The positive interaction was assessed by the investigating the expression of reporter genes. SD-Trp/-Leu denotes the synthetically defined medium which lacks tryptophan and leucine, SD-Trp/-Leu/+Aba/+X- $\alpha$-Gal denotes synthetically defined medium which lacks tryptophan and leucine but contains Aureobasidin A and X- $\alpha$-Gal and SD-Trp/-Leu/-His/-Ade/+Aba/+X- $\alpha$-Gal denotes synthetically defined medium which lacks tryptophan, leucine, histidine, and adenine but contains Aureobasidin A and X- $\alpha$-Gal. (B) HEK293 cells were co-transfected with Myc-PRMT5 and GFP or GFP-FAM47E constructs. 
FAM47E in HEK293 cells. We observed that the overexpression of FAM47E did not affect the PRMT5-MEP50 interaction suggesting that FAM47E interacts with PRMT5 and MEP50 without affecting the PRMT5-MEP50 complex (Fig S2B). Collectively, the results obtained using different approaches, establish FAM47E as an interaction partner of PRMT5.

\section{FAM47E enhances the stability of PRMT5 protein}

Because PRMT5 interacts with FAM47E, we investigated if PRMT5 methylates FAM47E. For this, we overexpressed GFP-FAM47E and the known PRMT5 substrate, GFP-SmD3, as a positive control, in HEK293 cells in the presence and absence of PRMT5 inhibitor, EPZ015666 (Chan-Penebre et al, 2015). GFP-FAM47E and GFP-SmD3 proteins were immunoprecipitated from these cells and their methylation status was investigated using pan symmetric dimethyl arginine antibody (SYM10). We observed a strong methylation signal in SmD3 protein, and this signal was reduced in the SmD3 protein isolated from the cells treated with EPZ015666. However, we could not detect any methylation signal in FAM47E protein (Fig S3). These findings suggest that FAM47E is an interaction partner of PRMT5 and is unlikely to be its substrate.

Protein-protein interaction may result in alteration of (i) protein stability of the interacting proteins (ii) functional outcomes or (iii) both. We first tested if PRMT5-FAM47E interaction alters the stability of PRMT5 and/or FAM47E. To investigate this, we overexpressed GFPtagged FAM47E and Myc-tagged PRMT5 individually or in combination. Whereas the overexpression of PRMT5 did not alter the levels of FAM47E, the overexpression FAM47E increased the PRMT5 protein levels (Fig S4). We next tested for the effect of FAM47E perturbation on the endogenous levels of PRMT5. For this, we depleted FAM47E levels by siRNA or overexpressed the GFP-tagged FAM47E in HEK293 cells and quantified the levels of PRMT5 protein in these cells by immunoblotting. We confirmed the knockdown of FAM47E by qRT-PCR (Fig S5) and immunoblotting (Fig 2B). We found that the overexpression of FAM47E increased the levels of PRMT5 protein by $\sim 2.2-$ fold (Fig 2A) and the depletion of FAM47E reduced levels of PRMT5 protein significantly by $\sim 39 \%$ (Fig 2 B). This suggests that FAM47E regulates the steady state levels of PRMT5 in HEK293 cells.

FAM47E might regulate the levels of PRMT5 by (i) increasing the synthesis of PRMT5, (ii) stabilizing PRMT5 protein by binding to it and preventing its degradation or (iii) both. To gain mechanistic understanding on how FAM47E regulates PRMT5 protein levels, we first quantified the levels of PRMT5 transcripts in HEK293 cells upon perturbation of FAM47E by using qRT-PCR. We observed that the knockdown of FAM47E did not alter the levels of PRMT5 mRNA levels significantly (Fig S6A). This suggests that the reduction of PRMT5 protein levels upon the depletion of FAM47E is not due to the decrease in the transcription of PRMT5. Interestingly, overexpression of FAM47E did not increase the levels of PRMT5 mRNA levels but decreased it by $15 \%$ (Fig S6B). Contrarily, this suggests that the elevated levels of PRMT5 protein might reduce its own transcription through feedback inhibition. This could imply that the increase in the protein levels of PRMT5 upon overexpression of FAM47E could be due to increase in the protein stability of PRMT5. We hypothesized that FAM47E binding to PRMT5 enhances the stability of PRMT5 protein by inhibiting its proteasomal degradation. To test this, we quantified the PRMT5 levels in HEK293 cells upon overexpression or knockdown of FAM47E and treated with the proteasomal inhibitor, MG-132. We found that the treatment of MG-132 abolished the FAM47E dependent increase or decrease of PRMT5 levels suggesting that FAM47E inhibits the proteasome-mediated degradation of PRMT5 (Fig 2C and D). Taken together, these observations suggest that FAM47E interacts with PRMT5 and increases the stability of PRMT5 by preventing its proteasomal degradation.

Because the E3 ubiquitin ligase CHIP interacts with PRMT5 and promotes its proteasomal degradation through ubiquitination (Zhang et al, 2016), we investigated whether FAM47E-PRMT5 interaction affects the binding of the E3 ubiquitin ligase CHIP with PRMT5. For this, we co-expressed GFP or GFP-PRMT5 and Myc-tagged CHIP with or without HA tagged FAM47E. We observed that the overexpression of FAM47E did not affect the PRMT5-CHIP interaction but on the contrary the overexpression of FAM47E enhanced PRMT5-CHIP interaction mildly (Fig S7). The mild enhancement of PRMT5-CHIP interaction might be due to the increase in the protein levels of PRMT5 upon overexpression of FAM47E. This suggests that the stabilization of PRMT5 by FAM47E is not mediated by disrupting the PRMT5-CHIP interaction. However, this does not rule out the possibility that FAM47E-PRMT5 interaction might block the CHIPmediated polyubiquitination of PRMT5. Because PRMT5 is ubiquitinated at multiple lysine residues (Zhang et al, 2016), it is also possible that FAM47E-PRMT5 interaction might inhibit the polyubiquitination of PRMT5 mediated by as yet unknown E3 ubiquitin ligase(s) that targets PRMT5. These findings lay foundation for future investigations to delineate the mechanisms underlying FAM47E inhibition of the proteasomal degradation of PRMT5.

Co-immunoprecipitation (Co-IP) was performed using GFP-Trap and the bound fractions were probed with Myc antibody. About $0.5 \%$ of the whole cell lysates which were used in Co-IP were probed with Myc antibody or GFP antibody. (C) HEK293 cells were co-transfected with HA-FAM47E and GFP or GFP-PRMT5 constructs. Co-IP was performed using GFP-Trap and the bound fractions were probed with HA antibody. About $1 \%$ of the whole cell lysates which were used in Co-IP were probed with HA antibody or GFP antibody. (D) Endogenous PRMT5 interacts with GFP tagged FAM47E. HEK293 cells were transfected with GFP or GFP-FAM47E constructs. Co-IP was performed using GFP-Trap and the bound fractions were probed with PRMT5 antibody. About 2\% of the whole cell lysates which were used in immunoprecipitation were probed with PRMT5 antibody or GFP antibody. (E) PRMT5 interacts with FAM47E directly. GST pull-down assay was carried out using recombinant GST-FAM47E and His-RMT5 proteins. The bound fractions were probed with His antibody. About $4 \%$ of His-PRMT5 (middle blot) and 2\% of GST-FAM47E (lower blot), which were used in the pulldown assay, were resolved in SDS-PAGE and stained with coomassie blue dye. (F) PRMT5 interacts with FAM47E at their endogenous levels in HEK293 cells. The cell lysates were prepared from HEK293 cells and immunoprecipitations were performed using rabbit IgG or FAM47E antibody and the bound fractions were probed with PRMT5 antibody. About $2 \%$ of the whole cell lysates which were used in immunoprecipitation were probed with PRMT5 antibody or FAM47E antibody. (G) The cell lysates were prepared from HEK293 cells and immunoprecipitations were performed using rabbit IgG or PRMT5 antibody and the bound fractions were probed with FAM47E antibody. About $0.1 \%$ of the whole cell lysates which were used in immunoprecipitation were probed with PRMT5 antibody or FAM47E antibody.

Source data are available for this figure. 
A

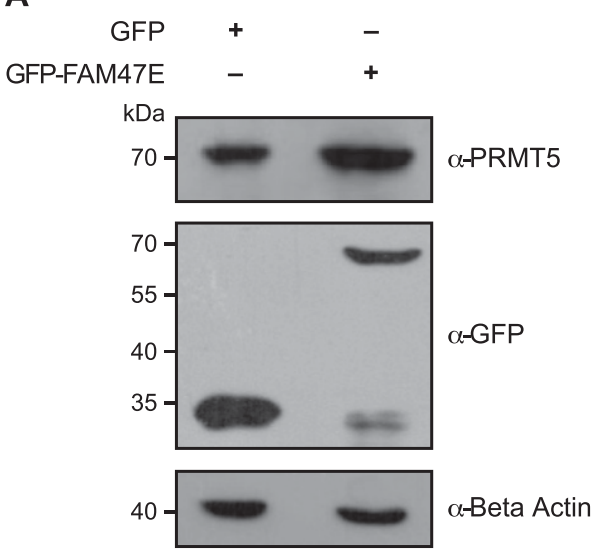

Relative levels of PRMT5

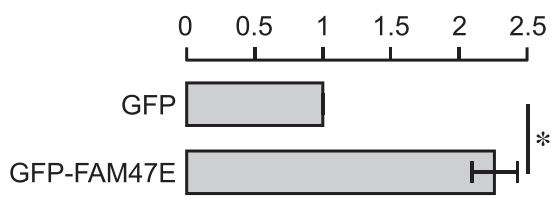

C

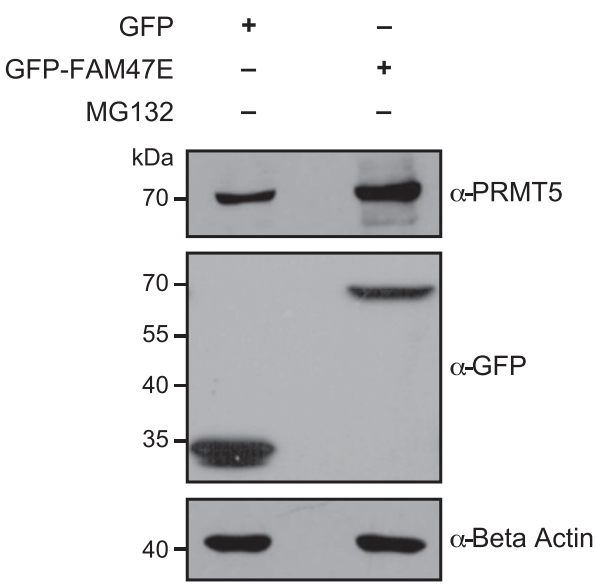

D
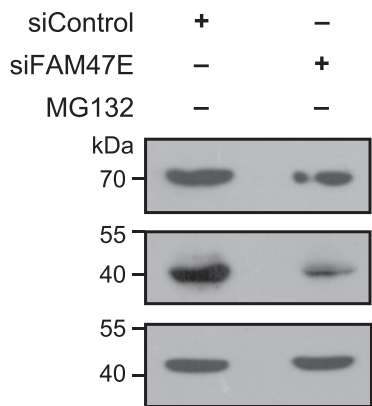

B

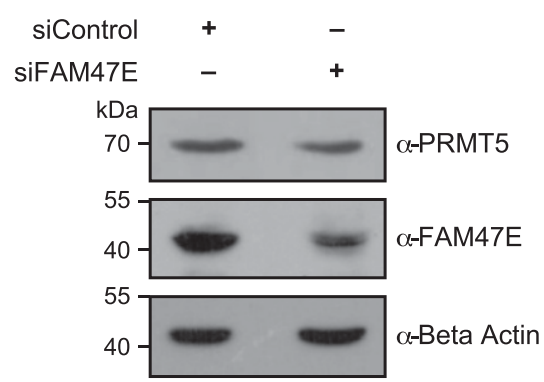

Relative levels of PRMT5
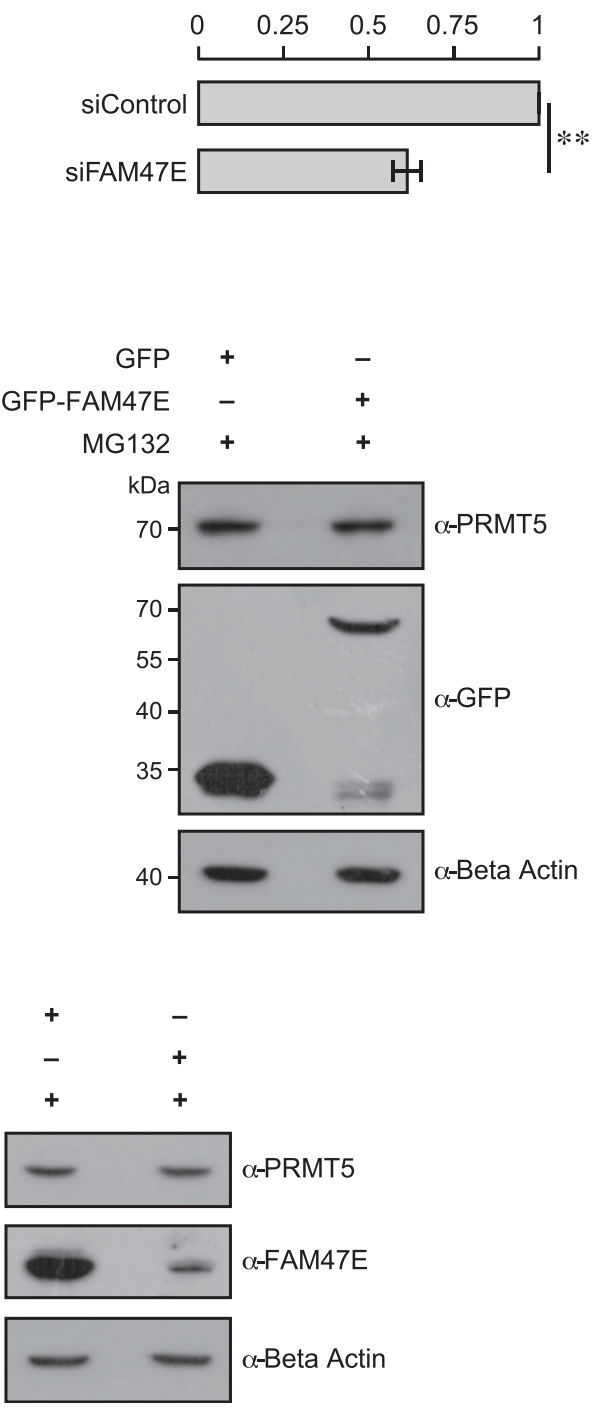

Figure 2. FAM47E increases the stability of PRMT5 protein.

(A) HEK293 cells were transfected with GFP vector or GFP-FAM47E construct. After $48 \mathrm{~h}$ of transfection, the cells were lysed, immunoblotted, and probed with PRMT5 antibody or GFP antibody or $\beta$ actin antibody (upper panel). The band intensities of PRMT5 and $\beta$ actin in the blots were quantified using Image software and the relative ratios of PRMT5 signal to $\beta$ actin signal are plotted in the graph (lower panel). The values represent the mean of three independent experiments, with error bars representing SD. Statistical significance was assessed using two-tailed $t$ test. * indicates $P<$

0.05. (B) HEK293 cells were transfected with control SiRNA vector or FAM47E siRNA. After $48 \mathrm{~h}$ of transfection, the cells were lysed, immunoblotted, and probed with PRMT5 antibody or FAM47E antibody or $\beta$ actin antibody (upper panel). The band intensities of PRMT5 and $\beta$ actin in the blots were quantified using Image) software and the relative ratios of PRMT5 signal to $\beta$ actin signal are plotted in the graph (lower panel). The values represent the mean of three independent experiments, with error bars representing SD.

Statistical significance was assessed using twotailed $t$ test. ${ }^{* *}$ indicates $P<0.01$. (C) HEK293 cells were transfected with GFP vector and GFP-FAM47E construct. After $40 \mathrm{~h}$ of transfection, the cells were treated with DMSO or MG-132 and incubated for $8 \mathrm{~h}$. The cells were lysed, immunoblotted and probed with PRMT5 antibody or GFP antibody or $\beta$ actin antibody. (D) HEK293 cells were transfected with control siRNA vector or FAM47E siRNA. After $40 \mathrm{~h}$ of transfection, the cells were treated with DMSO or MG-132 and incubated for $8 \mathrm{~h}$. The cells were lysed, immunoblotted, and probed with PRMT5 antibody or FAM47E antibody or $\beta$ actin antibody. 


\section{FAM47E promotes the chromatin association of PRMT5 and histone arginine methylation}

We next investigated whether FAM47E binding to PRMT5 affects the functionality of PRMT5. One of the well-established functions of PRMT5 is the epigenetic regulation of gene expression through histone arginine methylation. In this regard, we first tested if FAM47E binding affects the association of PRMT5 to chromatin. For this, we perturbed the FAM47E levels in HEK293 cells (Fig 3A) and isolated the soluble and chromatin fraction from these cells. We quantified the levels of PRMT5 in these fractions by immunoblotting. We found that the overexpression of FAM47E decreased the levels of PRMT5 in soluble fractions by 21\% (Fig 3B, left panel) and increased the abundance of PRMT5 in chromatin fractions dramatically by $\sim 2.2$-fold (Fig 3C, left panel) suggesting that FAM47E-PRMT5 interaction profoundly increases the association of PRMT5 to the chromatin. We observed that the knockdown of FAM47E increased the PRMT5 levels mildly in soluble fractions by $\sim 12 \%$ (Fig 3B, right panel) and decreased the PRMT5 level in chromatin fractions strongly by $\sim 33 \%$ (Fig 3C, right panel). These findings imply that FAM47E-PRMT5 interaction not only stabilizes PRMT5 but also enhances its association with the chromatin.

We next investigated if the increase in chromatin association of PRMT5 upon the overexpression of FAM47E translates into increased methylation of histones by PRMT5. To test this, we quantified the histone modifications that could be introduced by PRMT5 viz. (i) symmetric dimethylation of arginine 2 of histone 3 (H3R2me2s), (ii) symmetric dimethylation of arginine 8 of histone 3 (H3R8me2s), and (iii) symmetric dimethylation of arginine 3 of histone 4 (H4R3me2s) in histones isolated from HEK293 cells, upon overexpression or knockdown of FAM47E. We observed that the overexpression of FAM47E significantly increases the levels of H3R2me2s, H3R8me2s, and H4R3me2s modifications ( 2.1-folds; Fig $4 \mathrm{~A}$ and $\mathrm{C}$ ). Similarly, the depletion of FAM47E decreased the levels of PRMT5 mediated histone arginine methylation modifications by $\sim 40 \%$ (Fig 4B and D). These findings provide the functional significance of our observation that FAM47E regulates chromatin association of PRMT5 (Fig 3). Collectively, these observations suggest that (i) FAM47E is essential for the physiological epigenetic functions of PRMT5 and (ii) dysregulated levels of FAM47E might result in PRMT5-mediated detrimental effects.

FAM47E might reinforce the PRMT5-mediated epigenetic control of gene expression through histone arginine methylation. To investigate this, we perturbed the levels of FAM47E in HEK293 cells through overexpression or knockdown (Figs 5A and S8A) and quantified the expression of few well known PRMT5 target genes (Mongiardi et al, 2015; Sohail \& Xie, 2015) by quantitative PCR. We observed that overexpression of FAM47E significantly (i) reduces the expression of the carbamoyl-phosphate synthetase 2, aspartate transcarbamylase, and dihydroorotase (CAD) and cyclin D1 (CCND1) genes (Fig 5B), which are negatively regulated by PRMT5 (Mongiardi et al, 2015) and (ii) increases the expression of the doublecortin-like kinase 1 (DCLK1), pentraxin-related protein (PTX3), and tumor necrosis factor $\alpha$-induced protein 3 (TNFAIP3) genes (Fig 5B), which are positively regulated by PRMT5 (Sohail \& Xie, 2015). These results suggest that FAM47E overexpression mimics the PRMT5 overexpression in terms of epigenetic regulation of PRMT5-target genes.
To ensure that the effect of FAM47E overexpression on the regulation of PRMT5-target genes expression is mediated by PRMT5, we overexpressed FAM47E in PRMT5-depleted HEK293 cells and quantified the expression of PRMT5 target genes by qRT-PCR. The knockdown of PRMT5 in these cells was confirmed by qRT-PCR and immunoblotting (Figs 5A and S8B). We observed that the effect of FAM47E overexpression on the expression of PRMT5 target genes is lost in most cases or reversed in a few, in PRMT5-depleted cells, suggesting that FAM47E regulates the PRMT5 target genes expression through PRMT5 (Fig 5B). When FAM47E was depleted, there was an increase in the expression of the genes (CAD and CCND1) that are negatively regulated by PRMT5 and reduced expression of genes (DCLK1, PTX3, and TNFAIP3) that are known to be positively regulated by PRMT5, suggesting that FAM47E facilitates PRMT5mediated epigenetic regulation of gene expression (Fig 5B).

Because FAM47E regulates the PRMT5-mediated epigenetic regulation, we investigated the binding of FAM47E and PRMT5 at the promoters of the tested PRMT5 target genes by using chromatin immunoprecipitation (ChIP). The details about the promoter regions of these genes for ChIP analyses were obtained from previous studies (Khan et al, 2007; Oconnell et al, 2015; Rubino et al, 2017; Hermosilla et al, 2018; Lee et al, 2019). We found that both FAM47E and PRMT5 proteins were enriched at the promoter regions of the tested PRMT5 target genes. This indicates that FAM47E binds to the promoters of the PRMT5 target genes along with PRMT5 and contributes to PRMT5-mediated epigenetic regulation (Fig 5C). Taken together, these results establish that FAM47E tunes the PRMT5mediated epigenetic regulation of gene-expression by enhancing the association of PRMT5 with chromatin and subsequent histone arginine methylation modifications. Because FAM47E is distributed both in the cytoplasm and nucleus (Fig S9), we investigated whether FAM47E affects the non-chromatin functions of PRMT5. To address this, we analyzed the methylation levels of SmD3, a well-studied non-chromatin substrate of PRMT5 (Friesen et al, 2001; Meister et al, 2001) upon overexpression of FAM47E. For this, we overexpressed GFP-SmD3 with or without FAM47E-HA in HEK293 cells. GFP-SmD3 was immunoprecipitated from these cells and its methylation levels were assessed using pan symmetric dimethyl arginine antibody, SYM10. The reliability of SYM10 antibody in detecting PRMT5mediated methylation of SmD3 was confirmed by analyzing the methylation levels of SmD3 in the cells which were treated with or without PRMT5 inhibitor, EPZ015666 (Chan-Penebre et al, 2015) (Fig S3). We found that the overexpression of FAM47E did not alter methylation levels of SmD3 suggesting that FAM47E primarily affects the chromatin-associated epigenetic functions of PRMT5 (Fig S10). Nevertheless, this does not rule out the plausible role of FAM47E in affecting other non-chromatin functions of PRMT5. Further detailed investigations are required to unravel this aspect.

\section{FAM47E promotes cell proliferation and clonogenic capacity of the cells via PRMT5 axis}

PRMT5 levels are elevated in several cancer types and are associated with poor clinical outcomes (Bao et al, 2013; Győrffy et al, 2013; Stopa et al, 2015; Xiao et al, 2019). PRMT5 overexpression increases cell proliferation and colony forming capacity of the cells and the knockdown of PRMT5 reduces the cell proliferation and 
A
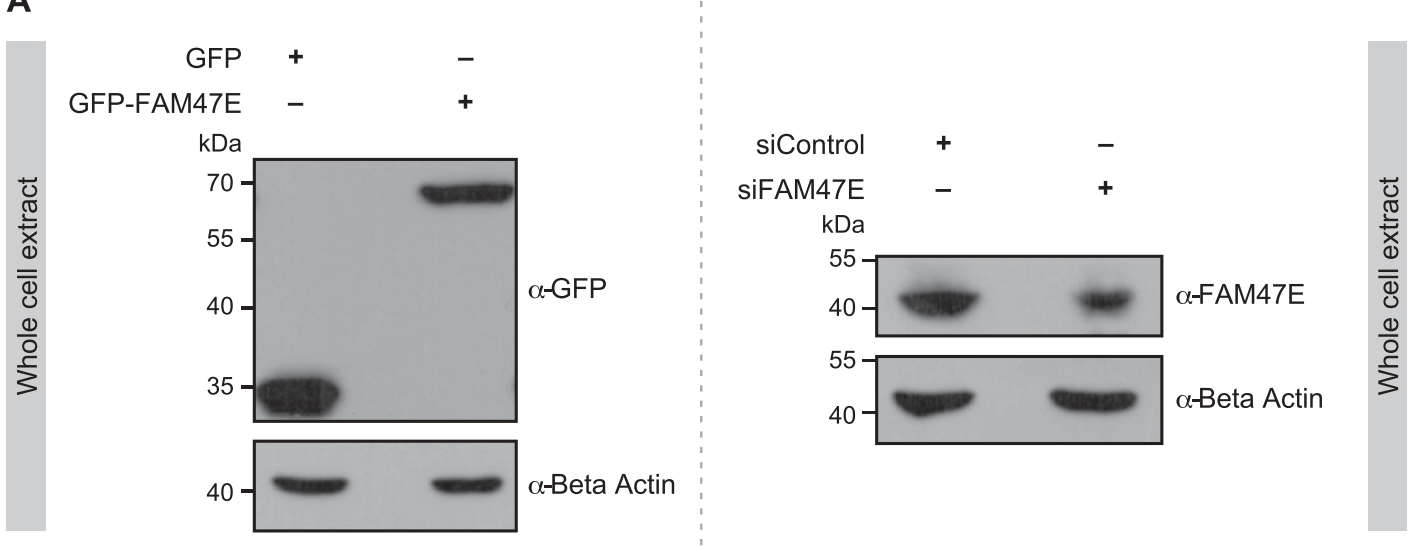

B
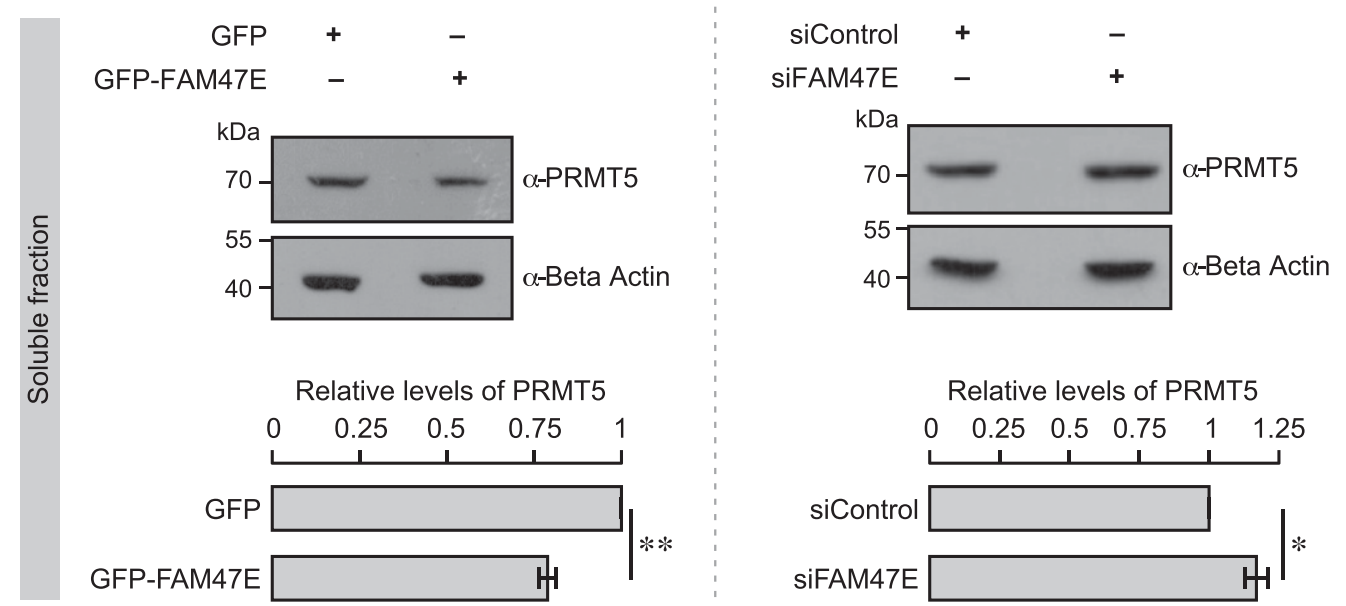

Relative levels of PRMT5
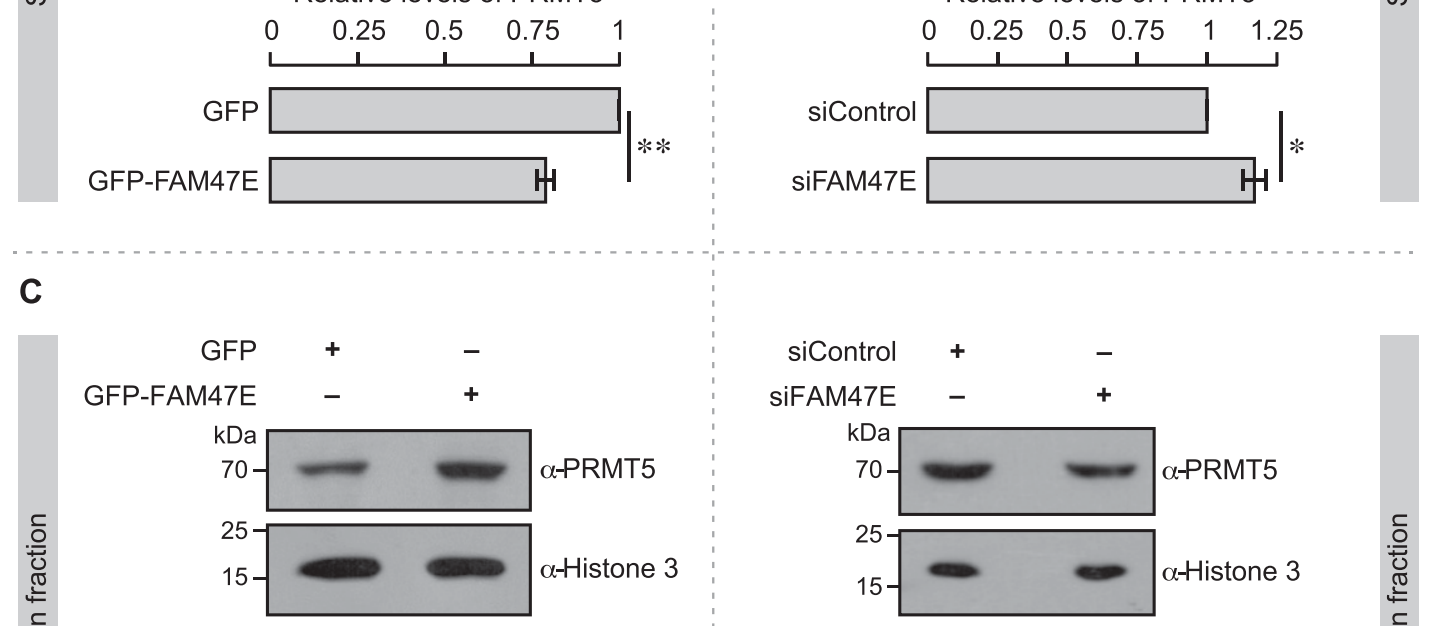

Relative levels of PRMT5

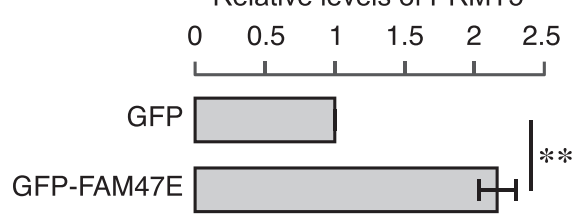

Relative levels of PRMT5

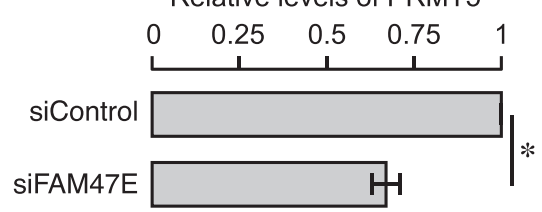

Figure 3. FAM47E enhances the chromatin association of PRMT5.

(A) HEK293 cells were transfected with, GFP vector or GFP-FAM47E construct or control siRNA or FAM47E siRNA. After $48 \mathrm{~h}$ of transfection, the cells were lysed, immunoblotted, and probed with GFP antibody or $\beta$ actin antibody (left panel) and FAM47E antibody or $\beta$ actin antibody (right panel). (B) HEK293 cells were transfected with GFP vector or GFP-FAM47E construct or control siRNA or FAM47E siRNA. After $48 \mathrm{~h}$ of transfection, the cells were lysed, the soluble fractions of the nuclei were isolated, and immunoblotting was performed using PRMT5 antibody or $\beta$ actin antibody (upper panels). The band intensities of PRMT5 and $\beta$ actin in the blots were quantified using Image) software and the relative ratios of PRMT5 signal to $\beta$ actin signal are plotted in the graph (lower panels). The values represent the mean of three independent 
A

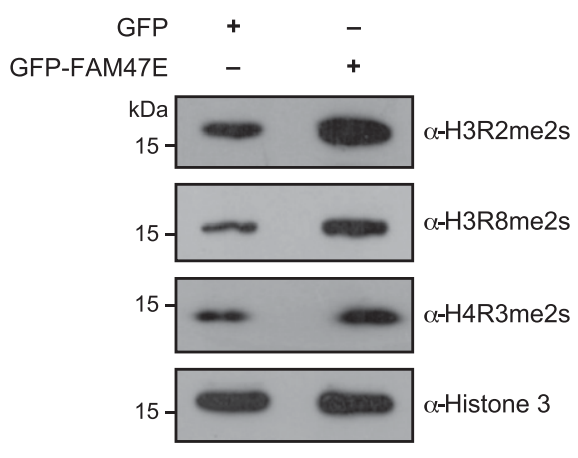

C

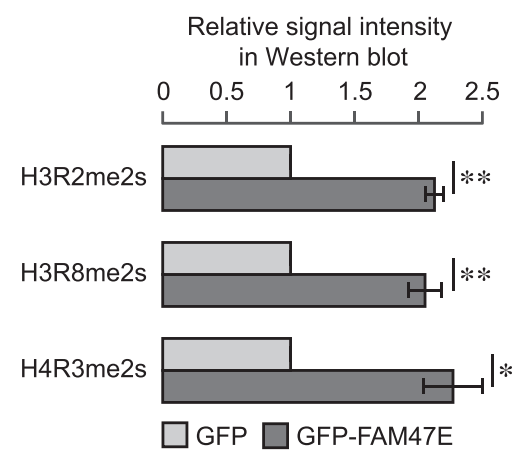

B

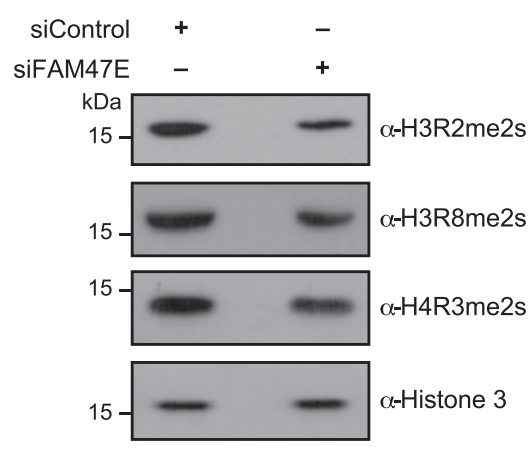

D

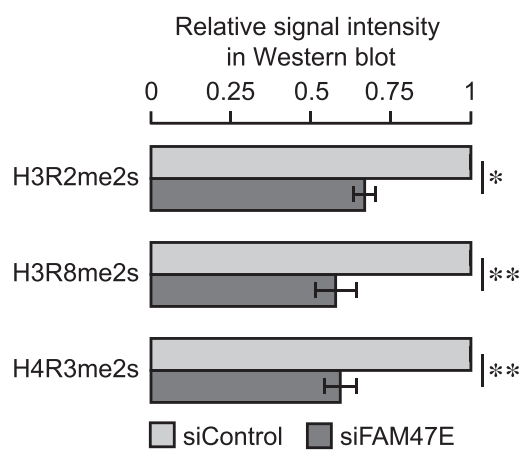

Figure 4. FAM47E promotes the histone methylation activity of PRMT5.

(A, B) HEK293 cells were transfected with GFP vector or GFP-FAM47E construct (A) or control siRNA or FAM47E SiRNA (B). The cells were harvested after $48 \mathrm{~h}$ of transfection and histones were isolated. The isolated histones were immunoblotted and probed with H3R2me2s antibody or H3R8me2s antibody or H4R3me2s antibody or histone 3 antibody. (C, D) The relative ratios of histone arginine methylation signals to histone 3 signal are plotted in the graph for FAM47E overexpression (C) and knockdown (D) conditions. The band intensities of histone arginine methylation modifications and histone 3 in the blots were quantified using ImageJ software. The values represent the mean of three independent experiments, with error bars representing standard deviations. Statistical significance was assessed using two-tailed $t$ test. * indicates $P<0.05$ and ** indicates $P<0.01$. colony forming capacity of the cells (Pal et al, 2004; Scoumanne et al, 2009; Wei et al, 2012; Stopa et al, 2015). Because FAM47E increases the PRMT5 protein levels and its chromatin association, we investigated the effect of FAM47E overexpression or knock down (Fig $6 \mathrm{~A}$ ) on cell proliferation and colony forming capacity of HeLa cells. The knock down of FAM47E in HeLa cells was confirmed by qRT-PCR and immunoblotting (Figs $6 \mathrm{~A}$ and S11A). The effect of FAM47E perturbation on cell proliferation was investigated by cell counting and MTT assays. We found that overexpression of FAM47E increased the cell proliferation and the depletion of FAM47E decreased the cell proliferation as reflected by the decrease or increase in doubling time, respectively (Figs 6B and S12). We observed similar results in the MTT assay as well (Fig 6C). We next probed the effect of FAM47E perturbation on the colony forming capacity of the HeLa cells. We observed that colony forming capacity of the cells increased by $45 \%$ upon the overexpression of FAM $47 E$ and decreased by $\sim 29 \%$ upon the knockdown of FAM47E (Fig 6D). These findings suggest that elevated levels of FAM47E can have oncogenic potential.

Based on our above observations, we hypothesized that increased cell proliferation and colony forming capacity upon FAM47E overexpression could be mediated through increase in PRMT5 levels/activity. To test if the effect of FAM47E overexpression on cell proliferation and clonogenic capacity is mediated by PRMT5, we overexpressed the FAM47E in PRMT5-depleted HeLa cells and investigated the cell proliferation and clonogenic capacity of the cells. The knockdown of PRMT5 in these cells was confirmed by qRTPCR and immunoblotting (Figs 6A and S11B). We observed that the effect of FAM47E overexpression on the cell proliferation and colony forming capacity is either lost or reduced in PRMT5-depleted cells suggesting that FAM47E increases the cell proliferation and colony forming capacity of the cells via PRMT5 axis (Fig 6B-D). Taken together, these data suggest that the FAM47E is important for cell proliferation mediated by PRMT5 and when dysregulated could have oncogenic potential.

\section{Discussion}

Genome wide association studies indicated that the FAM47E is associated with chronic kidney disease and Parkinson's disease (Ledo et al, 2015; Blauwendraat et al, 2019). However, nothing is known about the interaction partner or the function(s) of this protein. Here, we report that FAM47E interacts and regulates the

experiments, with error bars representing standard deviations. Statistical significance was assessed using two-tailed $t$ test. * indicates $P<0.05$ and $* *$ indicates $P<0.01$. (C) HEK293 cells were transfected with GFP vector or GFP-FAM47E construct or control siRNA or FAM47E siRNA. After 48 h of transfection, the cells were lysed, the chromatin fractions were prepared by benzonase digestion and immunoblotting was performed using PRMT5 antibody or histone 3 antibody (upper panels). The band intensities of PRMT5 and histone 3 in the blots were quantified using ImageJ software and the relative ratios of PRMT5 signal to histone 3 signal are plotted in the graph (lower panels). The values represent the mean of three independent experiments, with error bars representing standard deviations. Statistical significance was assessed using two-tailed $t$ test. * indicates $P<0.05$ and ${ }^{* *}$ indicates $P<0.01$. 
A
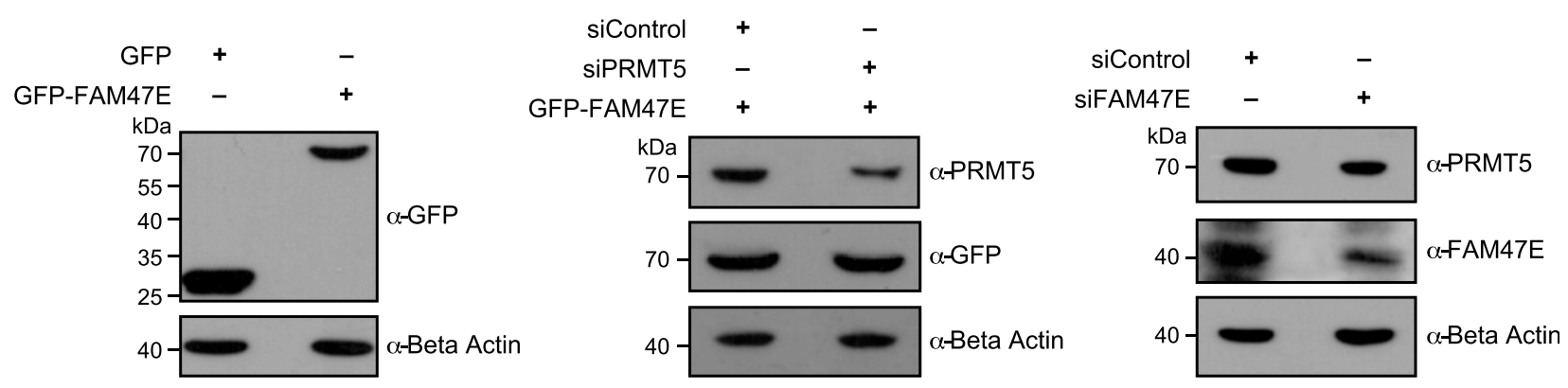

B

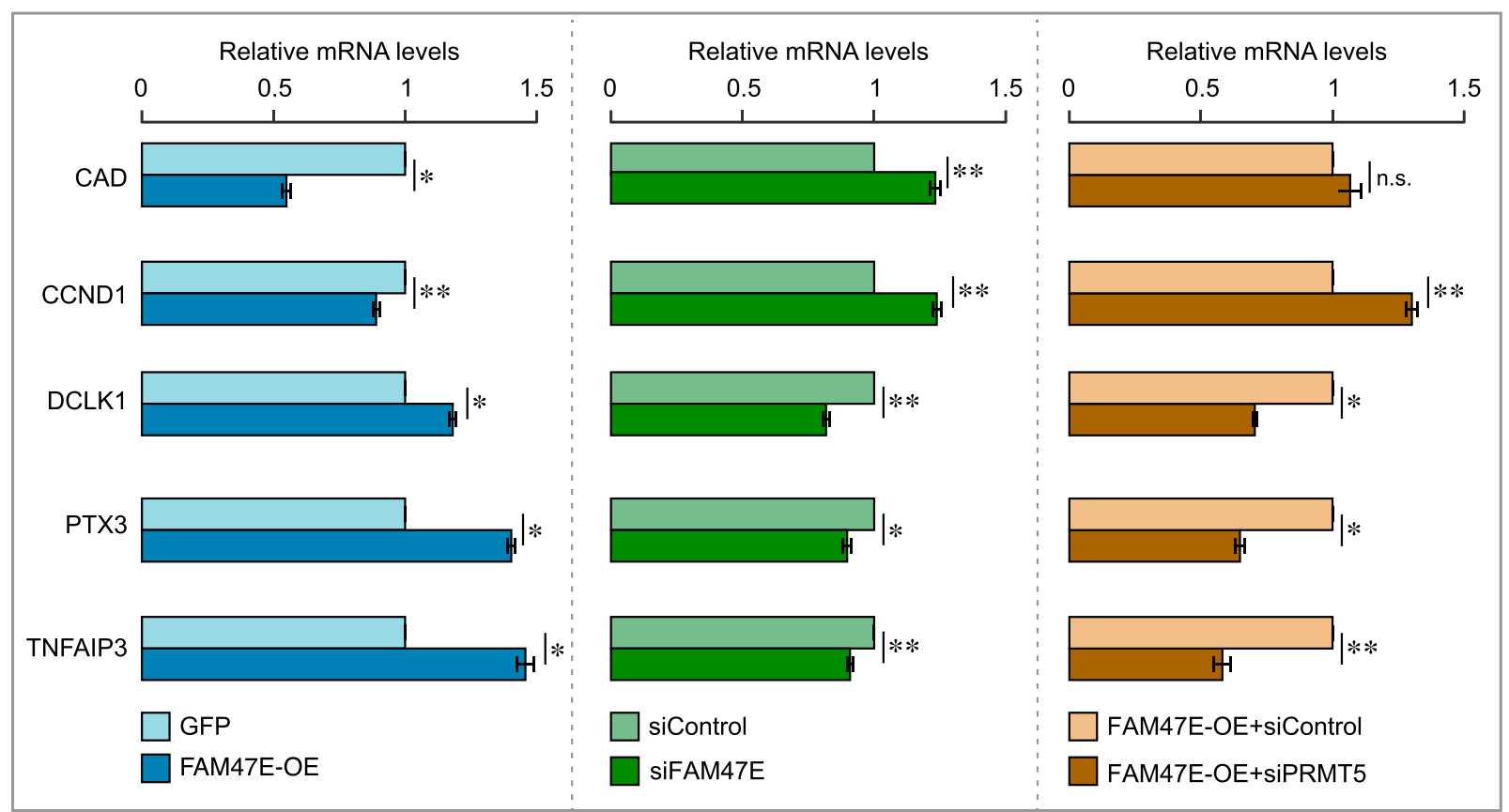

C

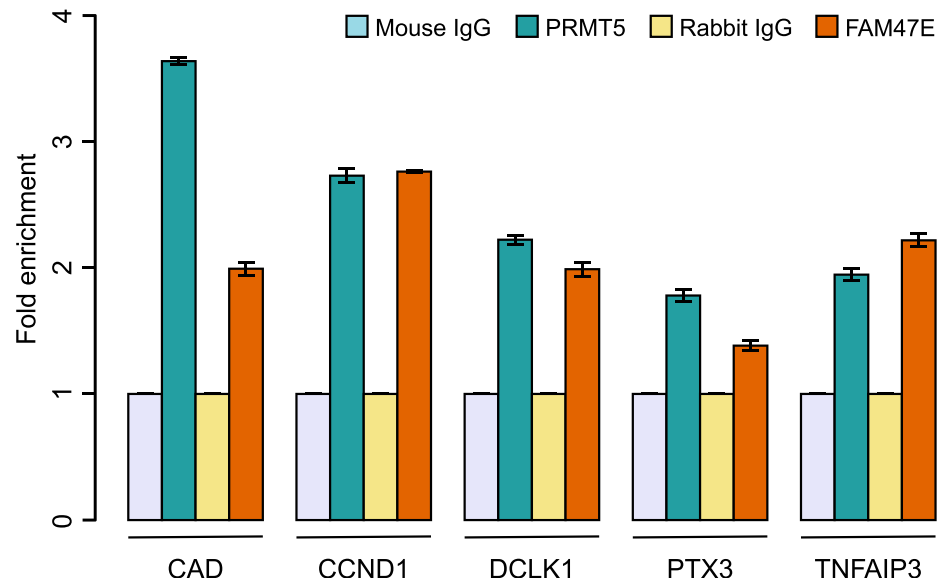

Figure 5. FAM47E regulates the expression of PRMT5 target genes.

(A, B) HEK293 cells were transfected with GFP vector or GFP-FAM47E construct or control siRNA or FAM47E siRNA or co-transfected with GFP-FAM47E construct and control siRNA or PRMT5 siRNA. After $48 \mathrm{~h}$ of transfections, the whole cell lysate and total RNA were isolated from these cells. (A) The cell lysates were immunoblotted and probed with GFP antibody or PRMT5 antibody or FAM47E antibody or $\beta$ actin antibody. (B) The transcripts levels of the indicated PRMT5 target genes in these cells were quantified by using quantitative RT-PCR. The mRNA levels of indicated PRMT5 target genes were normalized to GAPDH expression and are presented relative to the control sample. The values represent the mean of three independent experiments, with error bars representing standard deviations. Statistical significance was assessed using two-tailed $t$ test. * indicates $P<0.05$, ** indicates $P<0.01$, and n.s. indicates not significant. (C) The chromatin was prepared from HEK293 cells and the ChIP was performed using mouse IgG or PRMT5 antibody or rabbit IgG or FAM47E antibody. The association of PRMT5 and FAM47E with the promoters of PRMT5 target genes was investigated by analyzing the immunoprecipitated DNA using quantitative RT-PCR. Data are presented as fold enrichment relative to the control IgG binding and the values represent the mean of three independent experiments, with error bars representing standard deviations. 
A

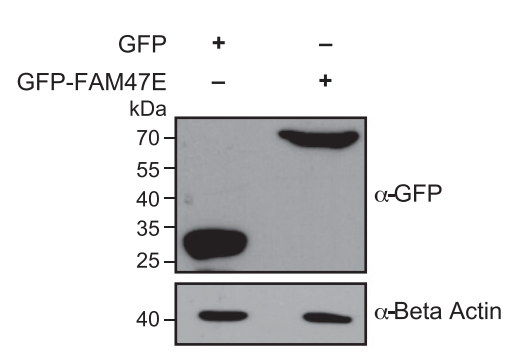

B

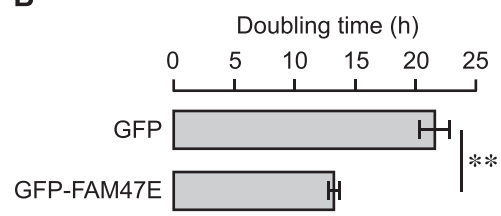

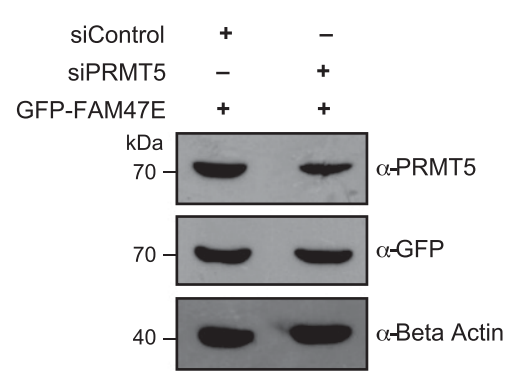

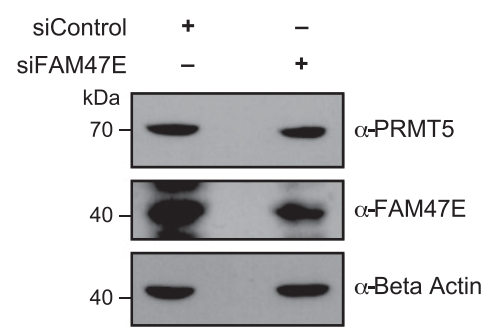

C
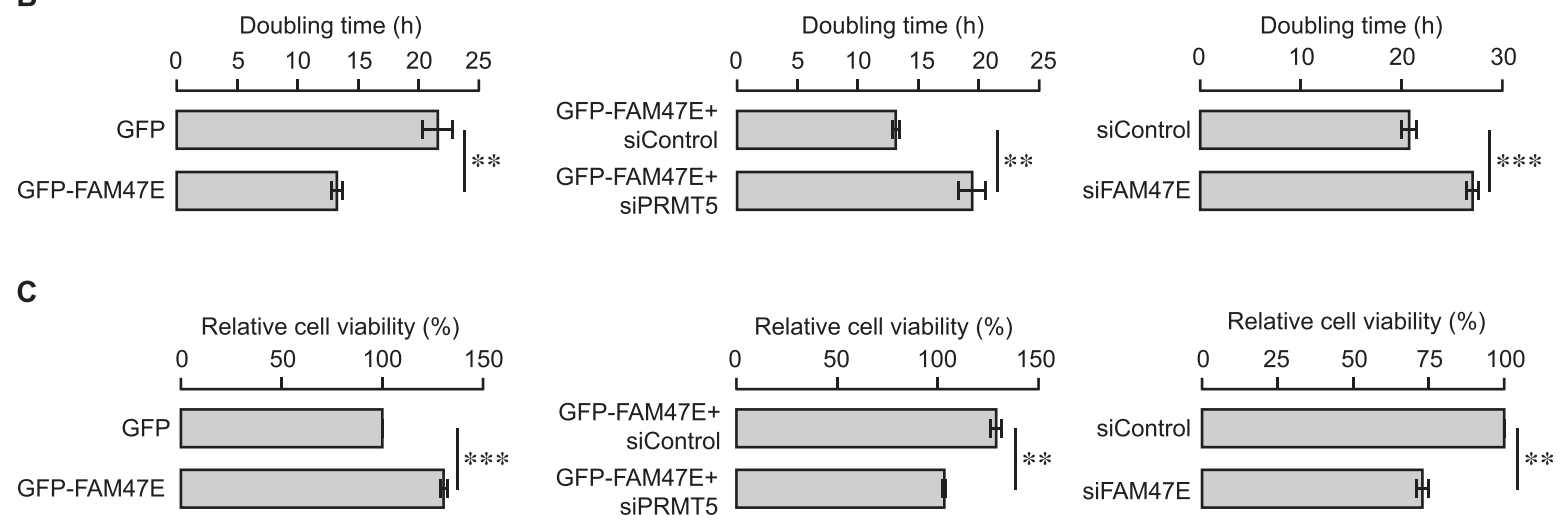

D

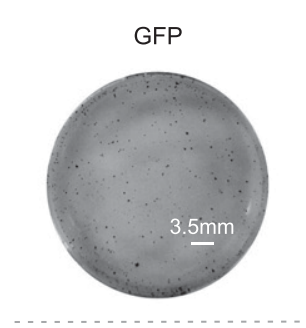

GFP-FAM47E+siControl

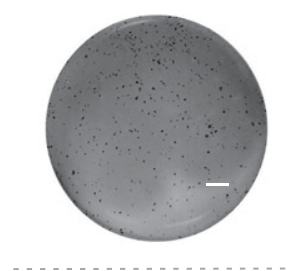

siControl

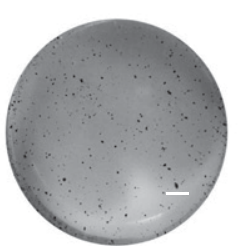

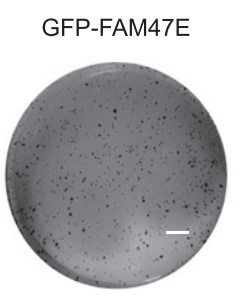

GFP-FAM47E+siPRMT5

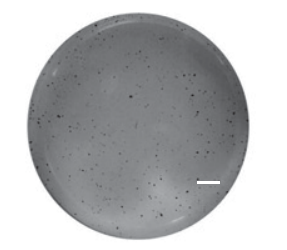

SiFAM47E

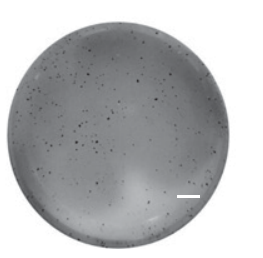

Relative colony forming potential
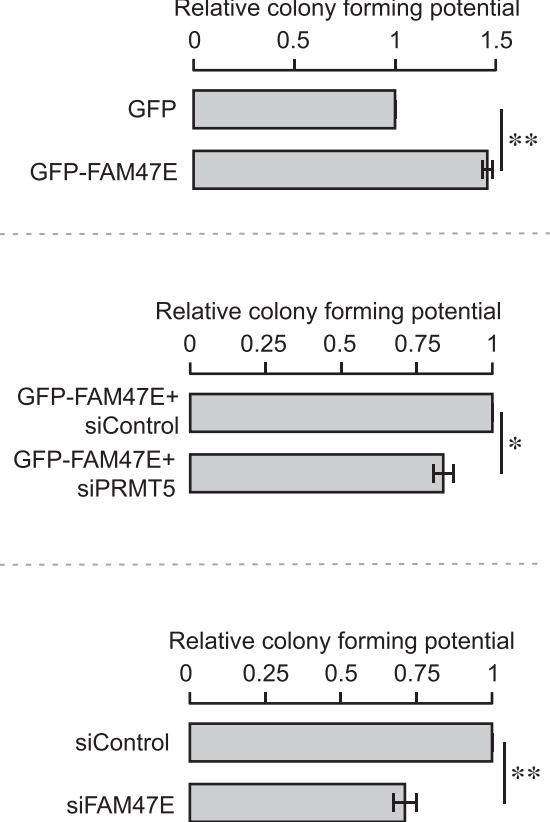

Figure 6. FAM47E increases cell proliferation and the clonogenic potential of HeLa cells through PRMT5.

(A) HeLa cells were transfected with GFP vector or GFP-FAM47E construct or control siRNA or FAM47E siRNA or co-transfected with GFP-FAM47E construct and control SiRNA or PRMT5 siRNA. After $48 \mathrm{~h}$ of transfection, the cells were lysed and probed with GFP antibody or PRMT5 antibody or FAM47E antibody or $\beta$ actin antibody. (B) HeLa cells were transfected with GFP vector or GFP-FAM47E construct or control siRNA or FAM47E siRNA or co-transfected with GFP-FAM47E construct and control siRNA or PRMT5 siRNA. The cells were counted after 48,72 and $96 \mathrm{~h}$ of post-transfection (Fig S12) and the doubling times were calculated. The values in the graph represent the mean of three independent experiments, with error bars representing SD. Statistical significance was assessed using two-tailed $t$ test. ${ }^{* *}$ indicates $P<0.01$ and ${ }^{* * *}$ indicates 
functions of the versatile arginine methyltransferase PRMT5 (Fig 7). These findings provide the first insights into the functional role(s) of FAM47E. On the other hand, several interaction partners of PRMT5 and their functional outcomes have been extensively studied. Specifically, MEP50 forms an octameric complex with PRMT5 and regulates its enzymatic activity and its levels (Friesen et al, 2002; Gonsalvez et al, 2007; Antonysamy et al, 2012; Ho et al, 2013; Saha \& Eckert, 2015; Saha et al, 2016; Chen et al, 2017). Strikingly, here we report that FAM47E increases the stability of PRMT5 and enhances its chromatin methylation activity (Fig 7). Although both the proteins seem to interact with PRMT5 and have overlapping effects, these could represent two distinct modes of regulation and function for PRMT5. For instance, here we show that FAM47E affects the stability of PRMT5 by inhibiting its proteasomal degradation. However, the mechanisms by which MEP50 regulates the levels of PRMT5 is unknown. In terms of functional impact, FAM47E enhances the chromatin methylation activity of PRMT5, by increasing its association with chromatin. On the other hand, MEP50 enhances the enzymatic activity of PRMT5 by increasing its affinity towards the substrate and the cofactor (Antonysamy et al, 2012). Further research is required to delineate the molecular mechanisms underlying the regulation and functional outcomes of PRMT5 upon binding with FAM47E and MEP50.

PRMT5 is a versatile protein which is involved in (i) epigenetic regulation via chromatin modifications and (ii) regulation of various other cellular processes through methylation and interaction of nonhistone proteins. We found that the FAM47E increases PRMT5mediated chromatin modifications by enhancing its association to the chromatin strongly and by decreasing the levels of PRMT5 in soluble fractions. This suggests that FAM47E promotes the epigenetic functions of PRMT5 and mitigates its non-epigenetic functions.

We also report that elevated levels of FAM47E could contribute the oncogenic properties of the cells as it increases the cell proliferation and colony forming capacity of the cells and demonstrate that the oncogenic functions of FAM47E is mediated by PRMT5. PRMT5 levels are up-regulated in several cancers and the depletion of PRMT5 reduces the carcinogenic properties of cells which makes the PRMT5 enzyme as an important therapeutic target for cancer therapy. This led to the discovery of selective inhibitors for PRMT5 enzyme and some of them entered clinical trials (Chan-Penebre et al, 2015; Bonday et al, 2018; Gerhart et al, 2018; Lin \& Luengo, 2019; Zhou et al, 2019). However, most of these inhibitors target the enzymatic activity of PRMT5 which affects wide range of functions that are mediated by PRMT5 in the cell. Our data demonstrate that FAM47E-PRMT5 interaction promotes the stability and epigenetic regulations of PRMT5. Interestingly, in conditions, especially in esophageal cancer, where PRMT5 is not up-regulated but FAM47E was significantly up-regulated, substantial proportion of targets positively regulated by PRMT5 were also significantly up-regulated (Fig S13). This suggests that FAM47E-mediated regulation of PRMT5 targets through stabilization of PRMT5 protein levels and increased association to chromatin could facilitate the enhanced expression of PRMT5 targets. This implies that the disruption of this interaction by small molecular inhibitors might serve as an alternative strategy for the preferential inhibition of PRMT5 epigenetic functions, which can be exploited as a specific therapy for the cancers in which PRMT5 mediated epigenetic signaling is dysregulated.

\section{Materials and Methods}

\section{Cloning, expression, and purification}

Using CDNA prepared from HEK293 cells, full-length FAM47E (NM_001242936.1, Isoform 2) and PRMT5 (NM_006109.4) sequences were PCR-amplified and cloned in different vectors. FAM47E was cloned in pGADT7 vector (Clontech) using EcoRI and BamHI sites, pGEX-6P2 vector (GE Healthcare) using BamHI and Xhol sites, and pCDNA3-EGFP vector (Invitrogen) using BamHI and EcoRI sites to generate pGADT7-FAM47E, pGEX-FAM47E, and pCDNA-GFP-FAM47E constructs, respectively. The oligo encoding the HA tag was introduced in to pCDNA4/myc-HisA vector (Invitrogen) using Xhol and Apal sites to generate pCDNA4-HA vector. FAM47E was also subcloned in pCDNA4-HA using BamHI and EcoRI sites to generate pCDNA4-HA-FAM47E construct. Similarly, full-length PRMT5 was cloned in PGBKT7 vector (Clontech) using EcoRI and BamHI sites, pCDN4/myc-HisA (Invitrogen) using EcoRI and Xhol sites, pET28a (Novagen) using BamHI and Xhol sites and pEGFP-C1 vector (Clontech) using Xhol and BamHI sites to generate pGBKT7-PRMT5, pCDNA4-Myc-PRMT5, pET28-PRMT5, and pEGFP-PRMT5 constructs, respectively. The sequence encoding the full length MEP50 (NM_024102.4) and the E3 ubiquitin ligase CHIP (NM_005861.4) was cloned in pCDN4/Myc-HisA vector using BamHI and Xhol sites to generate pCDNA4-Myc-MEP50 and pCDNA4-Myc-CHIP constructs, respectively. The sequence encoding the full-length SmD3 (NM_004175.5) was cloned in DEGFP-C1 vector using EcoRI and BamHI sites to generate pEGFP-SmD3 construct. The bacterial expression and purification of GST-tagged FAM47E and His-tagged PRMT5 was performed as described previously (Verma et al, 2017; Awasthi et al, 2018).

\section{Y2H screening}

Yeast two hybrid (Y2H) screening was performed using Matchmaker Gold Yeast two Hybrid system (Clontech) as per the manufacturer's instructions. To screen the interaction partners for PRMT5, pGBKT7-PRMT5

$P<0.001$. (C) HeLa cells were transfected with GFP vector or GFP-FAM47E construct or control siRNA or FAM47E siRNA or co-transfected with GFP-FAM47E construct and control siRNA or PRMT5 siRNA. After $48 \mathrm{~h}$ of transfections, MTT assay was carried out. The values in the graph represent the mean of three independent experiments, with error bars representing standard deviations. Statistical significance was assessed using two-tailed $t$ test. ** indicates $P<0.01$ and ${ }^{* * *}$ indicates $P<0.001$. (D) HeLa cells were transfected with GFP vector or GFP-FAM47E construct or control siRNA or FAM47E siRNA or co-transfected with GFP-FAM47E construct and control siRNA or PRMT5 siRNA. The colony-forming capacities of these cells were analyzed by staining the cells with crystal violet after $10 \mathrm{~d}$ of transfection. Experiments were performed in triplicates and the representative images are provided in the left panel. The scale bar is depicted. The colony numbers were counted using ImageJ software. The values in the graph represent the mean of three independent experiments, with error bars representing SD (right panel). Statistical significance was assessed using two-tailed $t$ test. * indicates $P<0.05$ and ** indicates $P<0.01$ 

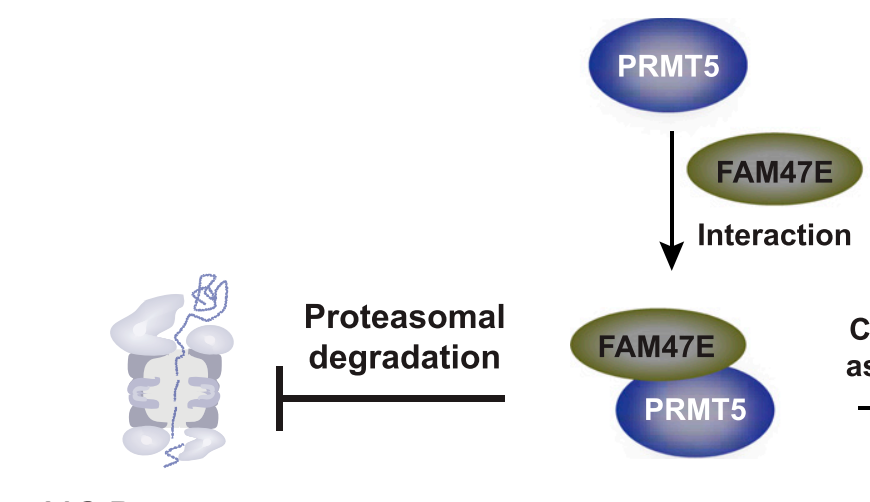

१H3R2me2s PH3R8me2s १H4R3me2s
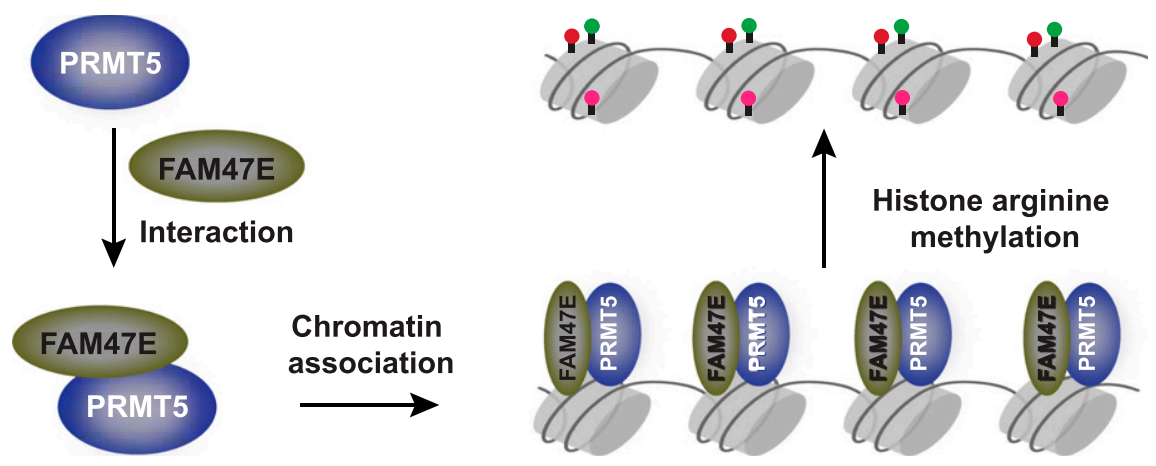

\section{S Proteasome}

Figure 7. Schema representing the regulatory role of FAM47E upon binding with PRMT5.

construct was used as a bait and human normalized cDNA library (Cat. no. 630480; Clontech) were used as prey. The normalized human cDNA library lacks the abundantly expressed transcripts, resulting in improved representation of low abundance cDNA. The positive interactions in $\mathrm{Y} 2 \mathrm{H}$ screening were identified by profiling the expression of reporter genes. The screening identified FAM47E as putative interaction partner for PRMT5.

The authenticity of the PRMT5-FAM47E interaction was investigated in $\mathrm{Y} 2 \mathrm{H}$ assay by co-transforming pGBKT7-PRMT5 with pGADT7 vector or pGADT7-FAM47E construct in MM Gold yeast strain (Clontech). Similarly, co-transformation was also carried out using pGADT7-FAM47E construct and pGBKT7 vector. The transformants were plated in a (i) synthetically defined medium which lacks tryptophan and leucine (SD-Trp/-Leu), (ii) synthetically defined medium which lacks tryptophan and leucine but contains Aureobasidin A and X- $\alpha$-Gal (SD-Trp/-Leu/+Aba/+X- $\alpha$-Gal) and (iii) synthetically defined medium which lacks tryptophan, leucine, histidine, and adenine but contains Aureobasidin A and $X-\alpha-G a l$ (SD-Trp/-Leu/-His/-Ade/+Aba/+X- $\alpha$-Gal) (Clontech). SD-Trp/-Leu medium allows the growth of all the co-transformants, whereas SD-Trp/-Leu/+Aba/+X- $\alpha$-Gal media allows the growth of Cotransformants which express the two reporter genes viz. AUR1-C and MEL1. Contrarily, SD-Trp/-Leu/-His/-Ade/+Aba/+X- $\alpha$-Gal medium selects the co-transformants that express the four reporter genes viz. HIS3, ADE2, AUR1-C, and MEL1. The expression of AUR1-C confers resistance to the toxic drug, Aureobasidin A, and the MEL1 codes for an enzyme, $\alpha$-galactosidase which acts on the chromogenic substrate $\mathrm{X}-\alpha$-Gal. The positive interactions were confirmed by assessing the expression of reporter genes.

\section{Cell-culture and transfection}

HEK293 and HeLa cells were purchased from National Centre for Cell Science, India and grown in DMEM (HiMedia) supplemented with $5 \%$ fetal bovine serum (HiMedia) and glutamine-penicillinstreptomycin solution (HiMedia). The cells were grown in incubator supplied with $5 \% \mathrm{CO}_{2}$. The plasmid constructs were transfected using standard calcium phosphate precipitation method. The
SiRNAs, siControl (siRNA Negative control, Cat. no. SR-CL000-005; Eurogentec), siFAM47E (SMARTpool - sigENOME siRNA targeting FAM47E, Cat. no. M185579-00-0005; Dharmacon), and siPRMT5 (5'CUU UGA GAC UGU GCU UUA U 3') were transfected using Lipofectamine 2000 Transfection Reagent (Thermo Fisher Scientific).

\section{Co-immunoprecipitation}

Co-Immunoprecipitation (Co-IP) experiments were performed to investigate the interaction of PRMT5 and FAM47E. For forward CO-IP, PCDNA4-Myc-PRMT5 construct was co-transfected with either pCDNA-GFP vector or pCDNA-GFP-FAM47E construct in HEK293 cells. For reverse Co-IP, pCDNA4-HA-FAM47E construct was cotransfected with either pEGFP vector or PEGFP-PRMT5 construct in HEK293 cells. After $48 \mathrm{~h}$ of transfection, the cells were harvested and lysed in RIPA buffer (50 mM Tris [pH 8.0], $150 \mathrm{mM} \mathrm{NaCl}, 0.5 \mathrm{mM}$ EDTA, 0.1\% NP40, 0.1\% SDS, 0.5\% deoxycholate, and protease inhibitor cocktail [Roche]). For co-immunoprecipitation, the cell lysates were incubated with GFP-Trap A beads (ChromoTek) for $8 \mathrm{~h}$ at $4^{\circ} \mathrm{C}$. After the incubation, the beads were washed extensively with RIPA buffer.

To investigate the interaction of FAM47E and MEP50, pCDNA-MycMEP50 construct was co-transfected with either PCDNA-GFP vector or pCDNA-GFP-FAM47E construct in HEK293 cells. To study the influence of FAM47E on PRMT5-MEP50 interaction, pCDNA-Myc-MEP50 construct was co-transfected with the combinations of pEGFP vector and PCDNA-HA vector or PEGFP-PRMT5 construct and PCDNA-HA vector or PEGFP-PRMT5 construct and PCDNA-HAFAM47E construct in HEK293 cells. To study the influence of FAM47E on PRMT5-CHIP interaction, pCDNA-Myc-CHIP construct was co-transfected with the combinations of pEGFP vector and $p C D N A-$ HA vector or PEGFP-PRMT5 construct and PCDNA-HA vector or PEGFP-PRMT5 construct and PCDNA-HA-FAM47E construct in HEK293 cells. After $48 \mathrm{~h}$ of transfection, the cells were harvested and lysed in lysis buffer (10 mM Tris, pH:7.5, $150 \mathrm{mM} \mathrm{NaCl}, 0.5 \mathrm{mM}$ EDTA, 0.5\% NP-40 and Protease Inhibitor Cocktail [Roche]). For coimmunoprecipitation, the cell lysates were incubated with GFPTrap A beads (ChromoTek) for $8 \mathrm{~h}$ at $4^{\circ} \mathrm{C}$. After the incubation, the 
beads were washed extensively with lysis buffer or wash buffer (10 $\mathrm{mM}$ Tris, pH:7.5, $300 \mathrm{mM} \mathrm{NaCl}, 0.5 \mathrm{mM}$ EDTA, 0.3\% NP-40, and protease inhibitor cocktail [Roche]).

The bound proteins were eluted by boiling the beads at $100^{\circ} \mathrm{C}$ for $5 \mathrm{~min}$, and the eluted proteins were separated in 12\% SDS-PAGE and transferred to PVDF membrane (GE Healthcare). The membrane was blocked overnight with blocking agent (GE Healthcare), washed with TTBS buffer (25 mM Tris, $150 \mathrm{mM} \mathrm{NaCl}$, and 0.1\% Tween 20) and probed with anti-Myc antibody (Cat. no. sc-40; Santa Cruz Biotechnology) or anti-HA antibody (Cat. no. 11867423001; Roche). The whole cell extract which was used in co-immunoprecipitation were immunoblotted and probed with anti-Myc antibody or anti-HA antibody or anti-GFP antibody (Cat. no. 632375; Clontech). The blots were developed using Super signal West Pico chemiluminescent substrate (Thermo Fisher Scientific) as per the manufacturer's instructions and the images were captured in X-ray sheets in darkroom.

To investigate the interaction of GFP-tagged FAM47E with endogenous PRMT5, HEK293 cells were transfected with either pCDNA-GFP vector or PCDNA-GFP-FAM47E construct. After $48 \mathrm{~h}$ of transfection the cells were lysed in RIPA buffer and immunporeciptation was carried out using GFP-Trap A beads (ChromoTek) as described above. The bound fractions were immunoblotted and probed with anti-PRMT5 antibody (Cat no. 07-405; Merck Millipore).

To investigate the interaction PRMT5 and FAM47E at their endogenous levels, HEK293 cells were lysed in a lysis buffer $(10 \mathrm{mM}$ Tris [pH 7.5], $150 \mathrm{mM} \mathrm{NaCl}, 0.5 \mathrm{mM}$ EDTA, 0.5\% NP40, and protease inhibitor cocktail). The lysates were incubated with rabbit IgG antibody (Cat. no. 2729; Cell Signaling Technology) or anti-FAM47E antibody (Cat. no. PA5-46681; Thermo Fisher Scientific) or antiPRMT5 antibody for $1 \mathrm{~h}$ at $4^{\circ} \mathrm{C}$. After the incubation, $30 \mu \mathrm{l}$ of Protein A Dynabeads (Invitrogen) were added to the lysates and incubated in rotator for $10 \mathrm{~h}$ at $4^{\circ} \mathrm{C}$. The beads were washed thrice with wash buffer (10 mM Tris [pH 7.5], $150 \mathrm{mM} \mathrm{NaCl}, 0.5 \mathrm{mM}$ EDTA, and $0.1 \% \mathrm{NP} 40$ ). The bound proteins were eluted by boiling the beads at $100^{\circ} \mathrm{C}$ for $5 \mathrm{~min}$. The eluted proteins were immunoblotted and probed with anti-FAM47E primary antibody or anti-PRMT5 antibody. The whole cell extract which was used in immunoprecipitation was also immunoblotted and probed with anti-FAM47E primary antibody or anti-PRMT5 antibody.

\section{GST pull-down assay}

Glutathione sepharose 4B (GE Healthcare) beads were coupled with either $50 \mu \mathrm{g}$ of GST protein or GST-tagged FAM47E protein in ice cold interaction buffer containing $20 \mathrm{mM}$ Hepes ( $\mathrm{pH}$ 7.5), $150 \mathrm{mM} \mathrm{KCl}, 0.2$ mM DTT, 1 mM EDTA, and 10\% glycerol. Then the beads were blocked with interaction buffer containing $5 \%$ bovine serum albumin for $1 \mathrm{~h}$ at $4^{\circ} \mathrm{C}$. The blocked beads were incubated with $25 \mu \mathrm{g}$ of His-tagged PRMT5 protein in binding buffer (10 mM Tris, $150 \mathrm{mM} \mathrm{NaCl}, 0.5 \mathrm{mM}$ EDTA, and $0.1 \% \mathrm{NP} 40$ ) for $3 \mathrm{~h}$ at $4^{\circ} \mathrm{C}$. After incubation the beads were washed thrice with wash buffer (10 mM Tris, $300 \mathrm{mM} \mathrm{NaCl}, 0.5 \mathrm{mM}$ EDTA, and $0.5 \%$ NP40). The bound proteins were eluted by boiling the beads with $2 \times$ LAP at $100^{\circ} \mathrm{C}$ for $5 \mathrm{~min}$. The eluted proteins were immunoblotted and probed with anti-His antibody (Cat. no. A00186100; GenScript).

\section{PRMT5 stability assay}

To investigate the stability of Myc-PRMT5 protein upon GFP-FAM47E overexpression or vice versa, HEK293 cells were transfected with pCDNA-GFP-FAM47E construct or pCDNA4-Myc-PRMT5 construct individually or in combination. The cells were collected $48 \mathrm{~h}$ of posttransfection and lysed in RIPA buffer. The lysates were immunoblotted and probed with anti-GFP antibody or anti-Myc antibody or anti-GAPDH antibody (Cat. no. MA5-15738; Thermo Fisher Scientific).

To study the effect of FAM47E perturbation on endogenous PRMT5 levels, HEK293 cells were transfected with control siRNA or FAM47E siRNA or pCDNA-GFP vector or pCDNA-GFP-FAM47E construct. After $40 \mathrm{~h}$ of transfection, the cells were treated with or without $50 \mu \mathrm{M}$ of MG-132 and incubated for $8 \mathrm{~h}$ and lysed in RIPA buffer. The lysates were immunoblotted and probed with antiPRMT5 antibody or anti-FAM47E antibody or anti-GFP antibody or anti- $\beta$ actin antibody (Cat. no. A2228; Sigma-Aldrich). The band intensities were quantified using the ImageJ software.

\section{Chromatin association studies}

The levels of PRMT5 association with the chromatin was investigated as described previously (Bian et al, 2015) with few modifications. Briefly, HEK293 cells were transfected with pCDNA-GFP vector or PCDNA-GFP-FAM47E construct and after $48 \mathrm{~h}$ of transfection, the cells were lysed in lysis buffer (10 mM Tris [pH 7.5], 150 $\mathrm{mM} \mathrm{NaCl}, 0.5 \%$ NP40, 0.5 mM EDTA, and Protease inhibitor cocktail). The lysates were centrifuged at $18,400 \mathrm{~g}$ for $10 \mathrm{~min}$ at $4^{\circ} \mathrm{C}$. The supernatant thus collected was labeled as soluble fraction and the pellet was resuspended in digestion buffer $(10 \mathrm{mM}$ Tris [pH 7.5], $150 \mathrm{mM} \mathrm{NaCl}, 0.5 \% \mathrm{NP} 40,1.5 \mathrm{mM} \mathrm{MgCl}$, protease inhibitor cocktail, and benzonase nuclease [Sigma-Aldrich]) and incubated in ice for $45 \mathrm{~min}$. The benzonase digestion was stopped by adding 2 mM EDTA, reaction mixtures were centrifuged at $21,100 \mathrm{~g}$ for 20 $\min$ at $4^{\circ} \mathrm{C}$ and the supernatants were collected and labeled as chromatin fractions. The soluble fractions were immunoblotted and probed with anti-PRMT5 antibody or anti- $\beta$ actin antibody and the chromatin fractions were immunoblotted and probed with anti-PRMT5 antibody or anti-histone 3 antibody (Cat. no. ab1791; Abcam). The band intensities were quantified using the ImageJ software.

\section{Investigation of histone arginine methylation modifications}

To investigate the effect of ectopic expression of FAM47E on histone arginine methylation modifications, HEK293 cells were transfected with PCDNA-GFP vector or pCDNA-GFP-FAM47E construct or control SiRNA or FAM47E siRNA. After $48 \mathrm{~h}$ of transfection, the cells were harvested and histones were isolated from these cells by standard acid extraction method as detailed previously (Shechter et al, 2007). The isolated histones were resolved in 16\% SDS-PAGE and immunoblotted and probed with anti-H4R3me2s antibody (Cat. no. ab5823; Abcam) or anti-H3R2me2s antibody (Cat. no. ab194684; Abcam) or anti-H3R8me2s (Cat. no. ab130740; Abcam) or antihistone 3 antibody (Cat. no. ab1791; Abcam). The band intensities were quantified using the ImageJ software. 
qRT-PCR analysis of PRMT5 target genes expression and FAM47E isoforms

HEK293 cells were transfected with pCDNA-GFP vector or pCDNA-GFPFAM47E construct or control siRNA or FAM47E siRNA or co-transfected with PCDNA-GFP-FAM47E construct and control siRNA or PRMT5 siRNA. After $48 \mathrm{~h}$ of post-transfection, the cells were harvested, total RNAs were extracted using Trizol reagent (Invitrogen) according to manufacturer's protocol and the cDNAs were prepared using Maxima H Minus Reverse Transcriptase (Thermo Fisher Scientific). The qRTPCR analyses were performed using SYBR green Master mix (Roche) as per manufacturer's protocol. The qRT-PCR reactions were performed in triplicates and each assay was repeated at least three times. The expression of target genes was normalized to the expression of GAPDH. To quantify the expression of different FAM47E isoforms, the total RNA was isolated from HEK293 cells and qRT-PCR analyses were performed as described above using isoform specific primers. The primers used in the qRT-PCR are listed in Table S1.

\section{Chromatin immunoprecipitation}

The chromatin was prepared from HEK293 cells as described previously (Awasthi et al, 2018). For the chromatin immunoprecipitation of PRMT5, $25 \mu \mathrm{g}$ of chromatin was incubated with $4 \mu \mathrm{g}$ of normal mouse IgG (Cat. no. sc-2025; Santa Cruz Biotechnology) or $4 \mu \mathrm{g}$ of antiPRMT5 antibody (Cat. no. sc-376937; Santa Cruz Biotechnology) for $2 \mathrm{~h}$ at $4^{\circ} \mathrm{C}$. After the incubation, $25 \mu \mathrm{l}$ of protein $\mathrm{G}$ beads were added to the tubes and incubated at $4^{\circ} \mathrm{C}$ with rotation for further $8 \mathrm{~h}$. For the chromatin immunoprecipitation of FAM47E, $25 \mu \mathrm{g}$ of chromatin was incubated with $4 \mu \mathrm{g}$ of normal rabbit IgG (Cat. no. 2729; Cell Signaling Technology) or $4 \mu \mathrm{g}$ of anti-FAM47E antibody (Cat. no. PA5-46681; Thermo Fisher Scientific) for $2 \mathrm{~h}$ at $4^{\circ} \mathrm{C}$. After the incubation, $25 \mu \mathrm{l}$ of protein $\mathrm{A}$ beads were added to the tubes and incubated at $4^{\circ} \mathrm{C}$ with rotation for further $8 \mathrm{~h}$. Then the beads were washed extensively and DNA was purified as detailed previously (Awasthi et al, 2018). The qRTPCR analyses of the immunoprecipitated DNA samples were carried out using SYBR green master mix (Roche) as described above. The primers used in the qRT-PCR are listed in Table S1.

\section{Methylation studies}

HEK293 cells were transfected with pEGFP-SmD3 construct or pCDNA-GFP-FAM47E construct and treated with or without PRMT5 inhibitor, EPZ015666 (Sigma-Aldrich) to investigate the methylation of FAM 47E by PRMT5. The pEGFP-SmD3 construct was co-transfected with PCDNA-HA vector or PCDNA-HA-FAM47E construct in HEK293 cells to study the influence of FAM47E on the methylation of SmD3 by PRMT5. After $48 \mathrm{~h}$ of transfection, the cells were lysed in the lysis buffer (10 mM Tris [pH:7.5] 150 mM NaCl, 0.5 mM EDTA, 0.5\% NP-40, and protease inhibitor cocktail; Roche) and incubated with GFPTrap A beads (ChromoTek) for $2 \mathrm{~h}$ at $4^{\circ} \mathrm{C}$. After the incubation, the beads were washed extensively with lysis buffer and bound fractions were eluted by boiling the beads at $100^{\circ} \mathrm{C}$ for 5 min and the eluted proteins were separated in 12\% SDS-PAGE, transferred to PVDF membrane and probed using or anti symmetric dimethyl arginine antibody, SYM10 (Cat. no. 07-412; Merck Millipore), or antiGFP antibody (Cat. no. 632375; Clontech).

\section{Immunofluorescence studies}

For immunofluorescence, HEK293 cells were grown on the coverslip up to $80 \%$ confluency. Then the cells were washed with PBS; fixed by using $4 \%$ formaldehyde and permeabilized with PBS containing $0.25 \%$ Triton X-100. Then the cells were incubated with anti-FAM47E antibody (Cat. no. PA5-46681; Thermo Fisher Scientific) for overnight at $4^{\circ} \mathrm{C}$ and probed with anti-rabbit IgG Dylight 633 antibody (Cat. no. 35563; Thermo Fisher Scientific). Then the cells were stained with DAPI and embedded using the Mowiol mounting medium, and the images were taken using the confocal microscope (LSM 510 Meta instrument).

\section{Cell proliferation and clonogenic assays}

HeLa cells were transfected with pCDNA-GFP vector or pCDNA-GFPFAM47E construct or control SIRNA or FAM47E SIRNA or COtransfected with pCDNA-GFP-FAM47E construct and control siRNA or PRMT5 siRNA. After 36, 48, 72 and 96 h of transfection, the cells were harvested, stained with trypan blue, and counted using a hemocytometer. MTT assays were performed as described previously (Awasthi et al, 2018).

HeLa cells were transfected with pCDNA-GFP vector or pCDNAGFP-FAM47E construct or control siRNA or FAM47E SIRNA or COtransfected with pCDNA-GFP-FAM47E construct and control siRNA or PRMT5 SiRNA. After $24 \mathrm{~h}$ of transfection, the clonogenic assay was carried out as described earlier (Awasthi et al, 2018).

\section{Supplementary Information}

Supplementary Information is available at https://doi.org/10.26508/lsa. 202000699.

\section{Acknowledgements}

This work was funded by Innovative Young Biotechnologist Award, Department of Biotechnology, Government of India (Grant No BT/03/IYBA/ 2010; A Dhayalan; Ramalingaswami Re-entry Fellowship: BT/RLF/Re-entry/ 05/2018; S Chavali; Research Associateship; RV Kadumuri), Board of Research in Nuclear Sciences (Grant No 37(1)/14/17/2017-BRNS/37019; A Dhayalan), Science \& Engineering Research Board (Grant No. SRG/2019/001785; S Chavali), Council of Scientific and Industrial Research (Junior and Senior Research Fellowships to B Chakrapani and S Awasthi), University Grants Commission (UGC), Government of India (Junior and Senior Basic Scientific Research Fellowships to MIK Khan and A Mahesh), Pondicherry University (PhD student fellowship to S Gupta and M Verma), Rajiv Gandhi Centre for Biotechnology Intramural grant (A Rajavelu), and IISER Tirupati (Post-doc fellowship to RV Kadumuri; Intramural support to S Chavali). We acknowledge Fund for Improvement of S\&T Infrastructure in Universities and Higher Educational Institutions Program of Department of Science and Technology (DST-FIST) and UGC-Special Assistant Programme that funded instrumentation facilities of Department of Biotechnology, Pondicherry University.

\section{Author Contributions}

B Chakrapani: conceptualization, formal analysis, validation, investigation, visualization, methodology, and writing-original draft. 
MIK Khan: conceptualization, formal analysis, validation, investigation, methodology, and writing-original draft.

RV Kadumuri: data curation, formal analysis, investigation, visualization, and writing-original draft.

S Gupta: validation, investigation, and methodology.

M Verma: validation, investigation, and methodology.

$S$ Awasthi: validation, investigation, and methodology.

G Govindaraju: validation and investigation.

A Mahesh: validation, investigation, and methodology.

A Rajavelu: conceptualization, investigation, and writing-original draft.

S Chavali: conceptualization, data curation, formal analysis, supervision, funding acquisition, validation, investigation, visualization, methodology, and writing-original draft, review, and editing. A Dhayalan: conceptualization, resources, formal analysis, supervision, funding acquisition, validation, investigation, visualization, methodology, project administration, and writing-original draft, review, and editing.

\section{Conflict of Interest Statement}

The authors declare that they have no conflict of interest.

\section{References}

Ancelin K, Lange UC, Hajkova P, Schneider R, Bannister AJ, Kouzarides T, Surani MA (2006) Blimp1 associates with Prmt5 and directs histone arginine methylation in mouse germ cells. Nat Cell Biol 8: 623-630. doi:10.1038/ncb1413

Andreu-Perez P, Esteve-Puig R, de Torre-Minguela C, López-Fauqued M, Bech-Serra JJ, Tenbaum S, Garcia-Trevijano ER, Canals F, Merlino G, Avila MA, et al (2011) Protein arginine methyltransferase 5 regulates ERK1/2 signal transduction amplitude and cell fate through CRAF. Sci Signal 4: ra58. doi:10.1126/scisignal.2001936

Antonysamy S, Bonday Z, Campbell RM, Doyle B, Druzina Z, Gheyi T, Han B, Jungheim LN, Qian Y, Rauch C, et al (2012) Crystal structure of the human PRMT5:MEP50 complex. Proc Natl Acad Sci U S A 109: 17960-17965. doi:10.1073/pnas.1209814109

Arribas-Layton M, Dennis J, Bennett EJ, Damgaard CK, Lykke-Andersen J (2016) The C-terminal RGG domain of human Lsm4 promotes processing body formation stimulated by arginine dimethylation. Mol Cell Biol 36: 2226-2235. doi:10.1128/mcb.01102-15

Awasthi S, Verma M, Mahesh A, Khan MIK, Govindaraju G, Rajavelu A, Chavali PL, Chavali S, Dhayalan A (2018) DDX49 is an RNA helicase that affects translation by regulating mRNA export and the levels of preribosomal RNA. Nucleic Acids Res 46: 6304-6317. doi:10.1093/nar/ gky231

Banasavadi-Siddegowda YK, Welker AM, An M, Yang X, Zhou W, Shi G, Imitola J, Li C, Hsu S, Wang J, et al (2018) PRMT5 as a druggable target for glioblastoma therapy. Neuro Oncol 20: 753-763. doi:10.1093/neuonc/ nox206

Bandyopadhyay S, Harris DP, Adams GN, Lause GE, McHugh A, Tillmaand EG, Money A, Willard B, Fox PL, DiCorleto PE (2012) HOXA9 methylation by PRMT5 is essential for endothelial cell expression of leukocyte adhesion molecules. Mol Cell Biol 32: 1202-1213. doi:10.1128/ mcb.05977-11

Bao X, Zhao S, Liu T, Liu Y, Liu Y, Yang X (2013) Overexpression of PRMT5 promotes tumor cell growth and is associated with poor disease prognosis in epithelial ovarian cancer. J Histochem Cytochem 61: 206-217. doi:10.1369/0022155413475452

Bedford MT, Clarke SG (2009) Protein arginine methylation in mammals: Who, what, and why. Mol Cell 33: 1-13. doi:10.1016/j.molcel.2008.12.013

Bian C, Chen Q, Yu X (2015) The zinc finger proteins ZNF644 and WIZ regulate the G9a/GLP complex for gene repression. Elife 4: e05606. doi:10.7554/ elife.08168

Blauwendraat C, Heilbron K, Vallerga CL, Bandres-Ciga S, von Coelln R, Pihlstrøm L, Simón-Sánchez J, Schulte C, Sharma M, Krohn L, et al (2019) Parkinson's disease age at onset genome-wide association study: Defining heritability, genetic loci, and $\alpha$-synuclein mechanisms. Mov Disord 34: 866-875. doi:10.1002/mds.27659

Bonday ZQ, Cortez GS, Grogan MJ, Antonysamy S, Weichert K, Bocchinfuso WP, Li F, Kennedy S, Li B, Mader MM, et al (2018) LLY-283, a potent and selective inhibitor of arginine methyltransferase 5, PRMT5, with antitumor activity. ACS Med Chem Lett 9: 612-617. doi:10.1021/ acsmedchemlett.8b00014

Branscombe TL, Frankel A, Lee JH, Cook JR, Yang Z, Pestka S, Clarke S (2001) PRMT5 (Janus kinase-binding protein 1) catalyzes the formation of symmetric dimethylarginine residues in proteins. J Biol Chem 276: 32971-32976. doi:10.1074/jbc.m105412200

Calabretta S, Vogel G, Yu Z, Choquet K, Darbelli L, Nicholson TB, Kleinman CL, Richard S (2018) Loss of PRMT5 promotes PDGFR degradation during oligodendrocyte differentiation and myelination. Dev Cell 46: 426-440.e5. doi:10.1016/j.devcel.2018.06.025

Cesaro E, De Cegli R, Medugno L, Florio F, Grosso M, Lupo A, Izzo P, Costanzo P (2009) The Kruppel-like zinc finger protein ZNF224 recruits the arginine methyltransferase PRMT5 on the transcriptional repressor complex of the aldolase A gene. J Biol Chem 284: 32321-32330. doi:10.1074/jbc.m109.043349

Chan-Penebre E, Kuplast KG, Majer CR, Boriack-Sjodin PA, Wigle TJ, Johnston LD, Rioux N, Munchhof MJ, Jin L, Jacques SL, et al (2015) A selective inhibitor of PRMT5 with in vivo and in vitro potency in MCL models. Nat Chem Biol 11: 432-437. doi:10.1038/nchembio.1810

Chen H, Lorton B, Gupta V, Shechter D (2017) A TGF $\beta$-PRMT5-MEP50 axis regulates cancer cell invasion through histone $\mathrm{H} 3$ and $\mathrm{H} 4$ arginine methylation coupled transcriptional activation and repression. Oncogene 36: 373-386. doi:10.1038/onc.2016.205

Chittka A, Nitarska J, Grazini U, Richardson WD (2012) Transcription factor positive regulatory domain 4 (PRDM4) recruits protein arginine methyltransferase 5 (PRMT5) to mediate histone arginine methylation and control neural stem cell proliferation and differentiation. J Biol Chem 287: 42995-43006. doi:10.1074/jbc.m112.392746

Cho E-C, Zheng S, Munro S, Liu G, Carr SM, Moehlenbrink J, Lu Y-C, Stimson L, Khan O, Konietzny R, et al (2012) Arginine methylation controls growth regulation by E2F-1. EMBO J 31: 1785-1797. doi:10.1038/emboj.2012.17

Chung J, Karkhanis V, Tae S, Yan F, Smith P, Ayers LW, Agostinelli C, Pileri S, Denis GV, Baiocchi RA, et al (2013) Protein arginine methyltransferase 5 (PRMT5) inhibition induces lymphoma cell death through reactivation of the retinoblastoma tumor suppressor pathway and polycomb repressor complex 2 (PRC2) silencing. J Biol Chem 288: 35534-35547. doi:10.1074/jbc.m113.510669

Clarke TL, Sanchez-Bailon MP, Chiang K, Reynolds JJ, Herrero-Ruiz J, Bandeiras TM, Matias PM, Maslen SL, Skehel JM, Stewart GS, et al (2017) PRMT5Dependent methylation of the TIP60 coactivator RUVBL1 is a key regulator of homologous recombination. Mol Cell 65: 900-916.e7. doi:10.1016/j.molcel.2017.01.019

Dacwag CS, Bedford MT, Sif S, Imbalzano AN (2009) Distinct protein arginine methyltransferases promote ATP-dependent chromatin remodeling function at different stages of skeletal muscle differentiation. Mol Cell Biol 29: 1909-1921. doi:10.1128/mcb.00742-08

Dacwag CS, Ohkawa Y, Pal S, Sif S, Imbalzano AN (2007) The protein arginine methyltransferase Prmt5 is required for myogenesis because it 
facilitates ATP-dependent chromatin remodeling. Mol Cell Biol 27: 384-394. doi:10.1128/mcb.01528-06

Friesen WJ, Paushkin S, Wyce A, Massenet S, Pesiridis GS, Van Duyne G, Rappsilber J, Mann M, Dreyfuss G (2001) The methylosome, a $20 \mathrm{~S}$ complex containing JBP1 and pICln, produces dimethylargininemodified Sm proteins. Mol Cell Biol 21: 8289-8300. doi:10.1128/ mcb.21.24.8289-8300.2001

Friesen WJ, Wyce A, Paushkin S, Abel L, Rappsilber J, Mann M, Dreyfuss G (2002) A novel WD repeat protein component of the methylosome binds Sm proteins. J Biol Chem 277: 8243-8247. doi:10.1074/jbc.m109984200

Gao G, Dhar S, Bedford MT (2017) PRMT5 regulates IRES-dependent translation via methylation of hnRNP A1. Nucleic Acids Res 45: 4359-4369. doi:10.1093/nar/gkw1367

Gerhart SV, Kellner WA, Thompson C, Pappalardi MB, Zhang X-P, Montes de Oca R, Penebre E, Duncan K, Boriack-Sjodin A, Le B, et al (2018) Activation of the p53-MDM4 regulatory axis defines the anti-tumour response to PRMT5 inhibition through its role in regulating cellular splicing. Sci Rep 8: 9711. doi:10.1038/s41598-018-28002-y

Gonsalvez GB, Tian L, Ospina JK, Boisvert FM, Lamond Al, Matera AG (2007) Two distinct arginine methyltransferases are required for biogenesis of Sm-class ribonucleoproteins. I Cell Biol 178: 733-740. doi:10.1083/ jcb.200702147

Guderian G, Peter C, Wiesner J, Sickmann A, Schulze-Osthoff K, Fischer U, Grimmler M (2011) RioK1, a new interactor of protein arginine methyltransferase 5 (PRMT5), competes with pICln for binding and modulates PRMT5 complex composition and substrate specificity. J Biol Chem 286: 1976-1986. doi:10.1074/jbc.m110.148486

Guo Z, Zheng L, Xu H, Dai H, Zhou M, Pascua MR, Chen QM, Shen B (2010) Methylation of FEN1 suppresses nearby phosphorylation and facilitates PCNA binding. Nat Chem Biol 6: 766-773. doi:10.1038/ nchembio. 422

Gurung B, Feng Z, Hua X (2013) Menin directly represses Gli1 expression independent of canonical Hedgehog signaling. Mol Cancer Res 11: 1215-1222. doi:10.1158/1541-7786.mcr-13-0170

Győrffy B, Surowiak P, Budczies J, Lánczky A (2013) Online survival analysis software to assess the prognostic value of biomarkers using transcriptomic data in non-small-cell lung cancer. PLoS One 8: e82241. doi:10.1371/journal.pone.0082241

Harris DP, Chandrasekharan UM, Bandyopadhyay S, Willard B, DiCorleto PE (2016) PRMT5-mediated methylation of NF-\$K\$B p65 at Arg174 is required for endothelial CXCL11 gene induction in response to TNF\$ $\alpha$ and IFN-\$y\$ costimulation. PLoS One 11: e0148905. doi:10.1371/ journal.pone.0148905

Hermosilla EV, Salgado G, Riffo E, Escobar D, Hepp MI, Farkas C, Galindo M, Morín V, García-Robles MA, Castro AF, et al (2018) SALL2 represses cyclins D1 and E1 expression and restrains G1/S cell cycle transition and cancer-related phenotypes. Mol Oncol 12: 1026-1046. doi:10.1002/ 1878-0261.12308

Ho M-C, Wilczek C, Bonanno JB, Xing L, Seznec J, Matsui T, Carter LG, Onikubo T, Kumar PR, Chan MK, et al (2013) Structure of the arginine methyltransferase PRMT5-MEP50 reveals a mechanism for substrate specificity. PLoS One 8: e57008. doi:10.1371/journal.pone.0057008

Hou Z, Peng H, Ayyanathan K, Yan K-P, Langer EM, Longmore GD, Rauscher FJ (2008) The LIM protein AJUBA recruits protein arginine methyltransferase 5 to mediate SNAIL-dependent transcriptional repression. Mol Cell Biol 28: 3198-3207. doi:10.1128/mcb.01435-07

Hsu J-M, Chen C-T, Chou C-K, Kuo H-P, Li L-Y, Lin C-Y, Lee H-J, Wang Y-N, Liu M, Liao H-W, et al (2011) Crosstalk between Arg 1175 methylation and Tyr 1173 phosphorylation negatively modulates EGFR-mediated ERK activation. Nat Cell Biol 13: 174-181. doi:10.1038/ncb2158

Huang J, Vogel G, Yu Z, Almazan G, Richard S (2011) Type II arginine methyltransferase PRMT5 regulates gene expression of inhibitors of differentiation/DNA binding Id2 and Id4 during glial cell differentiation. J Biol Chem 286: 44424-44432. doi:10.1074/ jbc.m111.277046

Jansson M, Durant ST, Cho E-C, Sheahan S, Edelmann M, Kessler B, La Thangue NB (2008) Arginine methylation regulates the p53 response. Nat Cell Biol 10: 1431-1439. doi:10.1038/ncb1802

Ju J, Wang Y, Liu R, Zhang Y, Xu Z, Wang Y, Wu Y, Liu M, Cerruti L, Zou F, et al (2014) Human fetal globin gene expression is regulated by LYAR. Nucleic Acids Res 42: 9740-9752. doi:10.1093/nar/gku718

Kanade SR, Eckert RL (2012) Protein arginine methyltransferase 5 (PRMT5) signaling suppresses protein kinase $\mathrm{C} \delta$ - and p38 $\delta$-dependent signaling and keratinocyte differentiation. J Biol Chem 287: 7313-7323. doi:10.1074/jbc.m111.331660

Khan S, Wu F, Liu S, Wu Q, Safe S (2007) Role of specificity protein transcription factors in estrogen-induced gene expression in MCF-7 breast cancer cells. J Mol Endocrinol 39: 289-304. doi:10.1677/jme-070043

Kwak YT, Guo J, Prajapati S, Park K-J, Surabhi RM, Miller B, Gehrig P, Gaynor RB (2003) Methylation of SPT5 regulates its interaction with RNA polymerase II and transcriptional elongation properties. Mol Cell 11: 1055-1066. doi:10.1016/s1097-2765(03)00101-1

Lacroix M, El Messaoudi S, Rodier G, Le Cam A, Sardet C, Fabbrizio E (2008) The histone-binding protein COPR5 is required for nuclear functions of the protein arginine methyltransferase PRMT5. EMBO Rep 9: 452-458. doi:10.1038/embor.2008.45

Ledo N, Ko Y-A, Park A-SD, Kang H-M, Han S-Y, Choi P, Susztak K (2015) Functional genomic annotation of genetic risk loci highlights inflammation and epithelial biology networks in CKD. J Am Soc Nephrol 26: 692-714. doi:10.1681/asn.2014010028

Lee J, Chan ST, Kim JY, Ou J-HJ (2019) Hepatitis C virus induces the ubiquitinediting enzyme A20 via depletion of the transcription factor upstream stimulatory factor 1 to support its replication. mBio 10: e01660-19. doi:10.1128/mbio.01660-19

Lin H, Luengo JI (2019) Nucleoside protein arginine methyltransferase 5 (PRMT5) inhibitors. Bioorg Med Chem Lett 29: 1264-1269. doi:10.1016/ j.bmcl.2019.03.042

Liu F, Zhao X, Perna F, Wang L, Koppikar P, Abdel-Wahab O, Harr MW, Levine RL, Xu H, Tefferi A, et al (2011) JAK2V617F-mediated phosphorylation of PRMT5 downregulates its methyltransferase activity and promotes myeloproliferation. Cancer Cell 19: 283-294. doi:10.1016/ j.ccr.2010.12.020

Liu L, Zhao X, Zhao L, Li J, Yang H, Zhu Z, Liu J, Huang G (2016) Arginine methylation of SREBP1a via PRMT5 promotes de novo lipogenesis and tumor growth. Cancer Res 76: 1260-1272. doi:10.1158/0008-5472.can-151766

Liu R, Gao J, Yang Y, Qiu R, Zheng Y, Huang W, Zeng Y, Hou Y, Wang S, Leng S, et al (2018) PHD finger protein 1 (PHF1) is a novel reader for histone H4R3 symmetric dimethylation and coordinates with PRMT5-WDR77/ CRL4B complex to promote tumorigenesis. Nucleic Acids Res 46: 6608-6626. doi:10.1093/nar/gky461

Lu X, Fernando TM, Lossos C, Yusufova N, Liu F, Fontán L, Durant M, Geng H, Melnick J, Luo Y, et al (2018) PRMT5 interacts with the BCL6 oncoprotein and is required for germinal center formation and lymphoma cell survival. Blood 132: 2026-2039. doi:10.1182/blood-201802-831438

Maloney A, Clarke PA, Naaby-Hansen S, Stein R, Koopman J-O, Akpan A, Yang A, Zvelebil M, Cramer R, Stimson L, et al (2007) Gene and protein expression profiling of human ovarian cancer cells treated with the heat shock protein 90 inhibitor 17-allylamino-17demethoxygeldanamycin. Cancer Res 67: 3239-3253. doi:10.1158/00085472.can-06-2968

Meister G, Eggert C, Bühler D, Brahms H, Kambach C, Fischer U (2001) Methylation of Sm proteins by a complex containing PRMT5 and the 
putative U snRNP assembly factor pICln. Curr Biol 11: 1990-1994. doi:10.1016/s0960-9822(01)00592-9

Migliori V, Müller J, Phalke S, Low D, Bezzi M, Mok WC, Sahu SK, Gunaratne J, Capasso P, Bassi C, et al (2012) Symmetric dimethylation of H3R2 is a newly identified histone mark that supports euchromatin maintenance. Nat Struct Mol Biol 19: 136-144. doi:10.1038/nsmb.2209

Mohr K, Koegl M (2012) High-throughput yeast two-hybrid screening of complex cDNA libraries. Methods Mol Biol 812: 89-102. doi:10.1007/ 978-1-61779-455-1_5

Mongiardi MP, Savino M, Bartoli L, Beji S, Nanni S, Scagnoli F, Falchetti ML, Favia A, Farsetti A, Levi A, et al (2015) Myc and omomyc functionally associate with the protein arginine methyltransferase 5 (PRMT5) in glioblastoma cells. Sci Rep 5: 15494. doi:10.1038/srep15494

Morettin A, Baldwin RM, Cote J (2015) Arginine methyltransferases as novel therapeutic targets for breast cancer. Mutagenesis 30: 177-189. doi:10.1093/mutage/geu039

Mounir Z, Korn JM, Westerling T, Lin F, Kirby CA, Schirle M, McAllister G, Hoffman G, Ramadan N, Hartung A, et al (2016) ERG signaling in prostate cancer is driven through PRMT5-dependent methylation of the Androgen receptor. Elife 5: e13964. doi:10.7554/elife.13964

Nagai Y, Ji MQ, Zhu F, Xiao Y, Tanaka Y, Kambayashi T, Fujimoto S, Goldberg MM, Zhang H, Li B, et al (2019) PRMT5 associates with the FOXP3 homomer and when disabled enhances targeted p185erbB2/neu tumor immunotherapy. Front Immunol 10: 174. doi:10.3389/ fimmu.2019.00174

Nordgård O, Dahle $\varnothing$, Andersen T $\varnothing$, Gabrielsen OS (2001) JAB1/CSN5 interacts with the GAL4 DNA binding domain: A note of caution about twohybrid interactions. Biochimie 83: 969-971. doi:10.1016/s0300-9084(01) 01329-3

Oconnell MR, Sarkar S, Luthra GK, Okugawa Y, Toiyama Y, Gajjar AH, Qiu S, Goel A, Singh P (2015) Epigenetic changes and alternate promoter usage by human colon cancers for expressing DCLK1-isoforms: Clinical implications. Sci Rep 5: 14983. doi:10.1038/srep14983

Pal S, Vishwanath SN, Erdjument-Bromage H, Tempst P, Sif S (2004) Human SWI/SNF-associated PRMT5 methylates histone $\mathrm{H} 3$ arginine 8 and negatively regulates expression of ST7 and NM23 tumor suppressor genes. Mol Cell Biol 24: 9630-9645. doi:10.1128/mcb.24.21.96309645.2004

Papatheodorou I, Moreno P, Manning J, Fuentes AM-P, George N, Fexova S, Fonseca NA, Füllgrabe A, Green M, Huang N, et al (2020) Expression Atlas update: From tissues to single cells. Nucleic Acids Res 48: D77-D83. doi:10.1093/nar/gkz947

Paul C, Sardet C, Fabbrizio E (2012) The histone- and PRMT5-associated protein COPR5 is required for myogenic differentiation. Cell Death Differ 19: 900-908. doi:10.1038/cdd.2011.193

Peng C, Wong CC (2017) The story of protein arginine methylation: Characterization, regulation, and function. Expert Rev Proteomics 14: 157-170. doi:10.1080/14789450.2017.1275573

Pollack BP, Kotenko SV, He W, Izotova LS, Barnoski BL, Pestka S (1999) The human homologue of the yeast proteins Skb1 and Hsl7p interacts with Jak kinases and contains protein methyltransferase activity. J Biol Chem 274: 31531-31542. doi:10.1074/jbc.274.44.31531

Ren J, Wang Y, Liang Y, Zhang Y, Bao S, Xu Z (2010) Methylation of ribosomal protein $S 10$ by protein-arginine methyltransferase 5 regulates ribosome biogenesis. J Biol Chem 285: 12695-12705. doi:10.1074/ jbc.m110.103911

Rubino M, Kunderfranco P, Basso G, Greco CM, Pasqualini F, Serio S, Roncalli M, Laghi L, Mantovani A, Papait R, et al (2017) Epigenetic regulation of the extrinsic oncosuppressor PTX3 gene in inflammation and cancer. Oncoimmunology 6: e1333215. doi:10.1080/2162402x.2017.1333215

Saha K, Adhikary G, Eckert RL (2016) MEP50/PRMT5 reduces gene expression by histone arginine methylation and this is reversed by PKCd/p38d signaling. J Invest Dermatol 136: 214-224. doi:10.1038/jid.2015.400
Saha K, Eckert RL (2015) Methylosome protein 50 and PKC $/$ p38 8 protein signaling control keratinocyte proliferation via opposing effects on p21Cip1 gene expression. J Biol Chem 290: 13521-13530. doi:10.1074/ jbc.m115.642868

Saloura V, Vougiouklakis T, Sievers C, Burkitt K, Nakamura Y, Hager G, van Waes C (2018) The role of protein methyltransferases as potential novel therapeutic targets in squamous cell carcinoma of the head and neck. Oral Oncol 81: 100-108. doi:10.1016/ j.oraloncology.2018.04.014

Scoumanne A, Zhang J, Chen X (2009) PRMT5 is required for cell-cycle progression and p53 tumor suppressor function. Nucleic Acids Res 37 4965-4976. doi:10.1093/nar/gkp516

Shechter D, Dormann HL, Allis CD, Hake SB (2007) Extraction, purification and analysis of histones. Nat Protoc 2: 1445-1457. doi:10.1038/ nprot.2007.202

Shin S-H, Lee GY, Lee M, Kang J, Shin H-W, Chun Y-S, Park J-W (2018) Aberrant expression of CITED2 promotes prostate cancer metastasis by activating the nucleolin-AKT pathway. Nat Commun 9: 4113. doi:10.1038/s41467-018-06606-2

Sohail M, Xie J (2015) Evolutionary emergence of a novel splice variant with an opposite effect on the cell cycle. Mol Cell Biol 35: 2203-2214. doi:10.1128/mcb.00190-15

Stopa N, Krebs JE, Shechter D (2015) The PRMT5 arginine methyltransferase: Many roles in development, cancer and beyond. Cell Mol Life Sci 72: 2041-2059. doi:10.1007/s00018-015-1847-9

Tamiya H, Kim H, Klymenko O, Kim H, Feng Y, Zhang T, Han JY, Murao A, Snipas SJ, Jilaveanu L, et al (2018) SHARPIN-mediated regulation of protein arginine methyltransferase 5 controls melanoma growth. J Clin Invest 128: 517-530. doi:10.1172/JCI95410

Tan CP, Nakielny S (2006) Control of the DNA methylation system component MBD2 by protein arginine methylation. Mol Cell Biol 26: 7224-7235. doi:10.1128/mcb.00473-06

Tee W-W, Pardo M, Theunissen TW, Yu L, Choudhary JS, Hajkova P, Surani MA (2010) Prmt5 is essential for early mouse development and acts in the cytoplasm to maintain ES cell pluripotency. Genes Dev 24: 2772-2777. doi:10.1101/gad.606110

Tsai W-C, Gayatri S, Reineke LC, Sbardella G, Bedford MT, Lloyd RE (2016) Arginine demethylation of G3BP1 promotes stress granule assembly. J Biol Chem 291: 22671-22685. doi:10.1074/jbc.m116.739573

Tsai W-W, Niessen S, Goebel N, Yates JR, Guccione E, Montminy M (2013) PRMT5 modulates the metabolic response to fasting signals. Proc Natl Acad Sci U S A 110: 8870-8875. doi:10.1073/pnas.1304602110

Verma M, Charles RCM, Chakrapani B, Coumar MS, Govindaraju G, Rajavelu A, Chavali S, Dhayalan A (2017) PRMT7 interacts with ASS1 and citrullinemia mutations disrupt the interaction. J Mol Biol 429: 2278-2289. doi:10.1016/j.jmb.2017.05.026

Wei T-YW, Juan C-C, Hisa J-Y, Su L-J, Lee Y-CG, Chou H-Y, Chen J-MM, Wu Y-C, Chiu S-C, Hsu C-P, et al (2012) Protein arginine methyltransferase 5 is a potential oncoprotein that upregulates G1 cyclins/cyclindependent kinases and the phosphoinositide 3-kinase/AKT signaling cascade. Cancer Sci 103: 1640-1650. doi:10.1111/j.13497006.2012.02367.x

Xiao W, Chen X, Liu L, Shu Y, Zhang M, Zhong Y (2019) Role of protein arginine methyltransferase 5 in human cancers. Biomed Pharmacother 114: 108790. doi:10.1016/j.biopha.2019.108790

Xu J, Xu X, Wang B, Ma Y, Zhang L, Xu H, Hu Y, Wu J, Cao X (2017) Nuclear carbonic anhydrase $6 \mathrm{~B}$ associates with PRMT5 to epigenetically promote IL-12 expression in innate response. Proc Natl Acad Sci U S A 114: 8620-8625. doi:10.1073/pnas.1700917114

Yang D, Liang T, Gu Y, Zhao Y, Shi Y, Zuo X, Cao Q, Yang Y, Kan Q (2016) Protein $\mathrm{N}$-arginine methyltransferase 5 promotes the tumor progression and radioresistance of nasopharyngeal carcinoma. Oncol Rep 35: 1703-1710. doi:10.3892/or.2015.4513 
Zhang H-T, Zeng L-F, He Q-Y, Tao WA, Zha Z-G, Hu C-D (2016) The E3 ubiquitin ligase CHIP mediates ubiquitination and proteasomal degradation of PRMT5. Biochim Biophys Acta 1863: 335-346. doi:10.1016/j.bbamcr.2015.12.001

Zhang S, Ma Y, Hu X, Zheng Y, Chen X (2019) Targeting PRMT5/Akt signalling axis prevents human lung cancer cell growth. J Cell Mol Med 23: 1333-1342. doi:10.1111/jcmm.14036

Zhao DY, Gish G, Braunschweig U, Li Y, Ni Z, Schmitges FW, Zhong G, Liu K, Li W, Moffat J, et al (2016) SMN and symmetric arginine dimethylation of RNA polymerase II C-terminal domain control termination. Nature 529: 48-53. doi:10.1038/nature16469

Zhou Z, Feng Z, Hu D, Yang P, Gur M, Bahar I, Cristofanilli M, Gradishar WJ, Xie $X-Q$, Wan $Y$ (2019) A novel small-molecule antagonizes PRMT5- mediated KLF4 methylation for targeted therapy. EBioMedicine 44: 98-111. doi:10.1016/j.ebiom.2019.05.011

Zhou Z, Sun X, Zou Z, Sun L, Zhang T, Guo S, Wen Y, Liu L, Wang Y, Qin J, et al (2010) PRMT5 regulates Golgi apparatus structure through methylation of the golgin GM130. Cell Res 20: 1023-1033. doi:10.1038/ cr.2010.56

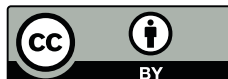

License: This article is available under a Creative Commons License (Attribution 4.0 International, as described at https://creativecommons.org/ licenses/by/4.0/). 